Proceedings of the Edinburgh Mathematical Society (2008) 51, 711-750 (C)

DOI:10.1017/S0013091506000150 Printed in the United Kingdom

\title{
SPECTRAL THEORY OF THE KLEIN-GORDON EQUATION IN KREIN SPACES
}

\author{
HEINZ LANGER ${ }^{1}$, BRANKO NAJMAN $^{2 *}$ AND CHRISTIANE TRETTER ${ }^{3}$ \\ ${ }^{1}$ Institut für Analysis und Scientific Computing, Technische Universität Wien, \\ Wiedner Hauptstrasse 8-10, 1040 Wien, Austria (hlanger@email.tuwien.ac.at) \\ ${ }^{2}$ Department of Mathematics, University of Zagreb, Bijenička 30, 41000 Zagreb, Croatia \\ ${ }^{3}$ Mathematisches Institut, Universität Bern, Sidlerstrasse 5, \\ 3012 Bern, Switzerland (tretter@math.unibe.ch)
}

(Received 19 January 2006)

Dedicated to the memory of Peter Jonas.

\begin{abstract}
In this paper the spectral properties of the abstract Klein-Gordon equation are studied. The main tool is an indefinite inner product known as the charge inner product. Under certain assumptions on the potential $V$, two operators are associated with the Klein-Gordon equation and studied in Krein spaces generated by the charge inner product. It is shown that the operators are self-adjoint and definitizable in these Krein spaces. As a consequence, they possess spectral functions with singularities, their essential spectra are real with a gap around 0 and their non-real spectra consist of finitely many eigenvalues of finite algebraic multiplicity which are symmetric to the real axis. One of these operators generates a strongly continuous group of unitary operators in the Krein space; the other one gives rise to two bounded semi-groups. Finally, the results are applied to the Klein-Gordon equation in $\mathbb{R}^{n}$.
\end{abstract}

Keywords: Klein-Gordon equation; Krein space; charge inner product; self-adjoint operators in Krein spaces; definitizable operators

2000 Mathematics subject classification: Primary 81Q05; 47B50

Secondary 47D03; 35Q40

\section{Introduction}

The Klein-Gordon equation

$$
\left(\left(\frac{\partial}{\partial t}-\mathrm{i} e q\right)^{2}-\Delta+m^{2}\right) \psi=0
$$

describes the motion of a relativistic spinless particle of mass $m$ and charge $e$ in an electrostatic field with potential $q$ (the velocity of light being normalized to 1 ); here $\psi$ is a complex-valued function of $x \in \mathbb{R}^{n}$ and of $t \in \mathbb{R}$.

If in (1.1) we replace the uniformly positive self-adjoint operator generated by the differential expression $-\Delta+m^{2}$ in the Hilbert space $L_{2}\left(\mathbb{R}^{n}\right)$ by a uniformly positive

\footnotetext{
* Sadly, Professor Branko Najman died in August 1996.
} 
self-adjoint operator $H_{0}$ in a Hilbert space $\mathcal{H}$ and the operator of multiplication with the function $e q$ by a symmetric operator $V$ in $\mathcal{H}$, we obtain the abstract Klein-Gordon equation

$$
\left(\left(\frac{\mathrm{d}}{\mathrm{d} t}-\mathrm{i} V\right)^{2}+H_{0}\right) u=0
$$

where $u$ is a function of $t \in \mathbb{R}$ with values in $\mathcal{H}$. Equation (1.2) can be transformed into a first-order differential equation for a vector function $\boldsymbol{x}$ in an appropriate space, formally given by $\mathcal{H} \oplus \mathcal{H}$, and a linear operator $A$ therein:

$$
\frac{\mathrm{d} \boldsymbol{x}}{\mathrm{d} t}=\mathrm{i} A \boldsymbol{x} .
$$

However, this is in general not possible with a self-adjoint operator $A$ in a Hilbert space.

In the literature two inner products have been associated with the Klein-Gordon equation (1.1), one of them representing the charge and the other one representing the energy of the particle. The energy inner product is used in numerous papers to study the spectral and scattering properties of (1.1), see, for example, $[\mathbf{5}, \mathbf{1 1}, \mathbf{1 2}, \mathbf{1 6}, \mathbf{2 0}, \mathbf{2 8}, \mathbf{2 9}, \mathbf{3 5}-\mathbf{3 7}$, $41,42,49,50]$. The charge inner product has been suggested by the physics literature (see [13]); it was first used in the pioneering work of Veselić [45-47] and subsequently in the papers $[\mathbf{3 4}, \mathbf{3 8}, \mathbf{4 8}]$, the unpublished manuscript* $[\mathbf{2 6}]$, as well as in $[\mathbf{9}, \mathbf{1 7}-\mathbf{1 9}]$. It is also called the number norm since it is related to the number operator in the theory of second quantization (see $[\mathbf{3}]$ ).

The reason for the preference of the energy inner product $\langle\cdot, \cdot\rangle$ may be that it is positive definite and generates a Hilbert space if the potential $V$ is small with respect to $H_{0}^{1 / 2}$; this can be seen from its formal definition

$$
\left\langle\boldsymbol{x}, \boldsymbol{x}^{\prime}\right\rangle=\left(\left(\begin{array}{cc}
H_{0}-V^{2} & 0 \\
0 & I
\end{array}\right) \boldsymbol{x}, \boldsymbol{x}^{\prime}\right)=\left(\left(H_{0}-V^{2}\right) x, x^{\prime}\right)+\left(y, y^{\prime}\right)
$$

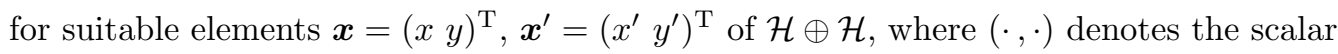
product in $\mathcal{H}$. The charge inner product $[\cdot, \cdot]$, however, is always indefinite: it is defined on elements $\boldsymbol{x}=\left(\begin{array}{ll}x y \\ y\end{array}\right)^{\mathrm{T}}, \boldsymbol{x}^{\prime}=\left(x^{\prime} y^{\prime}\right)^{\mathrm{T}}$ of $\mathcal{H} \oplus \mathcal{H}$ by a relation of the form

$$
\left[\boldsymbol{x}, \boldsymbol{x}^{\prime}\right]=\left(\left(\begin{array}{ll}
0 & I \\
I & 0
\end{array}\right) \boldsymbol{x}, \boldsymbol{x}^{\prime}\right)=\left(x, y^{\prime}\right)+\left(y, x^{\prime}\right) .
$$

Therefore, it is negative on an infinite-dimensional subspace (if $\mathcal{H}$ is infinite dimensional) and hence leads to a so-called Krein space. At first glance, these indefinite structures seem to be less convenient from the mathematical point of view. However, they allow deeper insight into the spectral properties of the Klein-Gordon equation, e.g. by providing a classification of the points of the spectrum into points of positive, negative or neutral type and sufficient conditions for the existence of corresponding strongly continuous groups of operators which are unitary with respect to the indefinite inner product (1.5).

* This manuscript, dating back to the late 1980s, was the starting point for the present paper and also for the papers $[\mathbf{1 8}, \mathbf{2 7}]$. 
In this paper we associate two operators, $A_{1}$ and $A_{2}$, with the abstract Klein-Gordon equation (1.2). Formally, both operators arise from the second-order differential equation (1.2) by means of the substitution

$$
x=u, \quad y=\left(-\mathrm{i} \frac{\mathrm{d}}{\mathrm{d} t}-V\right) u,
$$

which leads to a first-order differential equation (1.3) of the form

$$
\frac{\mathrm{d} \boldsymbol{x}}{\mathrm{d} t}=\mathrm{i}\left(\begin{array}{cc}
V & I \\
H_{0} & V
\end{array}\right) \boldsymbol{x}
$$

for the vector function $\boldsymbol{x}=(x y)^{\mathrm{T}}$. However, we consider the block operator matrix in (1.7) in two different Krein spaces induced by the charge inner product (1.5), and we prove the self-adjointness of the corresponding operators $A_{1}$ and $A_{2}$ in these Krein spaces.

The main results of this paper concern the structure and classification of the spectrum for $A_{1}$ and $A_{2}$, the existence of a spectral function with singularities, the generation of a strongly continuous group of unitary operators, the existence of solutions of the Cauchy problem for the abstract Klein-Gordon equation (1.2) and an application of these results to the Klein-Gordon equation (1.1) in $\mathbb{R}^{n}$. The main tools for this are techniques from the theory of block operator matrices and results from the theory of self-adjoint operators in Krein spaces.

The paper is organized as follows: in $\S 2$ we present some basic notation and definitions from spectral theory and we give a brief review of results from the theory of self-adjoint and definitizable operators in Krein spaces. In $\S 3$ we introduce the first operator, $A_{1}$, associated with (1.7), which acts in the Hilbert space $\mathcal{G}_{1}=\mathcal{H} \oplus \mathcal{H}$. Equipped with the charge inner product (1.5), the space $\mathcal{G}_{1}$ becomes a Krein space, which we denote by $\mathcal{K}_{1}$. The block operator matrix in (1.7) is formally symmetric with respect to the charge inner product $[\cdot, \cdot]$ since, for $\boldsymbol{x} \in\left(\mathcal{D}(V) \cap \mathcal{D}\left(H_{0}\right)\right) \oplus \mathcal{D}(V), \boldsymbol{x}^{\prime} \in \mathcal{H} \oplus \mathcal{H}$,

$$
\left[\left(\begin{array}{cc}
V & I \\
H_{0} & V
\end{array}\right) \boldsymbol{x}, \boldsymbol{x}^{\prime}\right]=\left(\left(\begin{array}{cc}
H_{0} & V \\
V & I
\end{array}\right) \boldsymbol{x}, \boldsymbol{x}^{\prime}\right) .
$$

We show that if $V$ is relatively bounded with respect to $H_{0}^{1 / 2}$, then the block operator matrix in (1.7) is essentially self-adjoint in $\mathcal{K}_{1}$, and we denote its self-adjoint closure by $A_{1}$. Using a certain factorization of $A_{1}-\lambda, \lambda \in \mathbb{C}$, we relate the spectral properties of $A_{1}$ to those of the quadratic operator polynomial $L_{1}$ in $\mathcal{H}$ given by

$$
L_{1}(\lambda)=I-\left(S-\lambda H_{0}^{-1 / 2}\right)\left(S^{*}-\lambda H_{0}^{-1 / 2}\right), \quad \lambda \in \mathbb{C},
$$

where $S$ is the bounded operator $S=V H_{0}^{-1 / 2}$.

In $\S 4$ we define the second operator, $A_{2}$, associated with (1.7) in the more complicated Hilbert space $\mathcal{G}_{2}=\mathcal{H}_{1 / 4} \oplus \mathcal{H}_{-1 / 4}$; here $\mathcal{H}_{\alpha},-1 \leqslant \alpha \leqslant 1$, is a scale of Hilbert spaces induced by the fractional powers $H_{0}^{\alpha}$ of the uniformly positive operator $H_{0}$. We equip $\mathcal{G}_{2}$ 
with the charge inner product (1.5), where now the brackets on the right-hand side of (1.5) denote the duality

$$
(x, y)=\left(H_{0}^{\alpha} x, H_{0}^{-\alpha} y\right), \quad x \in \mathcal{H}_{\alpha}, y \in \mathcal{H}_{-\alpha},
$$

between the spaces $\mathcal{H}_{\alpha}$ and $\mathcal{H}_{-\alpha}$ for $\alpha=\frac{1}{4}$ and $\alpha=-\frac{1}{4}$; the corresponding space $\mathcal{K}_{2}$ is a Krein space. An analogue of formula (1.8) in $\mathcal{H}_{1 / 4} \oplus \mathcal{H}_{-1 / 4}$ shows that the block operator matrix in (1.7), now considered as an operator in $\mathcal{G}_{2}$, is formally symmetric with respect to the charge inner product $[\cdot, \cdot]$. If the resolvent set $\rho\left(L_{1}\right)$ is non-empty, we associate a self-adjoint operator $A_{2}$ in the Krein space $\mathcal{K}_{2}$ with the block operator matrix in (1.7).

The operator $A_{2}$ was first considered by Veselić [45] (see also $[\mathbf{3 4}, \mathbf{4 6}, \mathbf{4 7}]$ ). He showed that, under his assumptions, $A_{2}$ is similar to a self-adjoint operator in a Hilbert space. The operators $A_{1}$ and $A_{2}$ were also introduced by Jonas [18] using so-called range restriction (see [17]). In [18] and in $[\mathbf{4 5}]$ it was supposed that $V H_{0}^{-1 / 2}$ is compact. The operator $A_{2}$ was also studied in $[\mathbf{9}, \mathbf{3 4}]$ for the cases when either $V H_{0}^{-1 / 2}$ is compact or $\left\|V H_{0}^{-1 / 2}\right\|<1$. The main results of the present paper are proved under the more general condition

$$
S:=V H_{0}^{-1 / 2}=S_{0}+S_{1}, \quad\left\|S_{0}\right\|<1, S_{1} \text { compact. }
$$

Under this assumption, in $\S 5$, we study and compare the spectral properties of the operators $A_{1}$ and $A_{2}$. We show that $A_{1}$ and $A_{2}$ are definitizable (for the definition of definitizability see $\S 2$ ), that their spectra, essential spectra and point spectra coincide and are symmetric to the real axis, that their essential spectra are real and have a gap around 0, and that the non-real spectrum consists of a finite number of complex conjugate pairs of eigenvalues of finite algebraic multiplicity; this number is bounded by the number $\kappa$ of negative eigenvalues of the operator $I-S^{*} S$ in $\mathcal{H}$. As a consequence of the definitizability, the operators $A_{1}$ and $A_{2}$ possess spectral functions with at most finitely many singularities.

At the end of $\S 5$ we compare the results for $A_{1}$ with results for another operator associated with the Klein-Gordon equation in [27]. This operator, $A$, arises from the second-order differential equation (1.2) by means of the substitution

$$
x=u, \quad y=-\mathrm{i} \frac{\mathrm{d} u}{\mathrm{~d} t},
$$

which leads to a first-order differential equation of the form

$$
\frac{\mathrm{d} \boldsymbol{x}}{\mathrm{d} t}=\mathrm{i}\left(\begin{array}{cc}
0 & I \\
H_{0}-V^{2} & 2 V
\end{array}\right) \boldsymbol{x} .
$$

The operator $A$, formally given by the block operator matrix in (1.11), acts in the space $\mathcal{G}=\mathcal{H}_{1 / 2} \oplus \mathcal{H}$; it is defined if $V$ is $H_{0}^{1 / 2}$-bounded and $1 \in \rho\left(S^{*} S\right)$. These two assumptions guarantee that a self-adjoint operator $H=H_{0}^{1 / 2}\left(I-S^{*} S\right) H_{0}^{1 / 2}$ can be associated with the entry $H_{0}-V^{2}$ in (1.11). Under the additional assumption 1.9 , the space $\mathcal{K}$ is a Pontryagin space and $A$ is a self-adjoint (and hence definitizable) operator in $\mathcal{K}$. We 
prove that the spectra, essential spectra and point spectra of $A$ and of $A_{1}$ (and hence also of $A_{2}$ ) coincide.

In $\S 6$ we show that $A_{2}$ generates a strongly continuous group $\left(\mathrm{e}^{\mathrm{i} t A_{2}}\right)_{t \in \mathbb{R}}$ of unitary operators in the Krein space $\mathcal{K}_{2}$. Hence, the Cauchy problem

$$
\frac{\mathrm{d} \boldsymbol{x}}{\mathrm{d} t}=\mathrm{i} A_{2} \boldsymbol{x}, \quad \boldsymbol{x}(0)=\boldsymbol{x}_{0},
$$

has a unique classical solution given by

$$
\boldsymbol{x}(t)=\mathrm{e}^{\mathrm{i} t A_{2}} \boldsymbol{x}_{0}, \quad t \in \mathbb{R},
$$

if $\boldsymbol{x}_{0} \in \mathcal{D}\left(A_{2}\right)$; if only $\boldsymbol{x}_{0} \in \mathcal{K}_{2}$, then (1.13) is a mild solution of (1.12). To this end, we prove that $\infty$ is a regular critical point of $A_{2}$, thus generalizing a result in [9] for the special cases $S_{0}=0$ and $S_{1}=0$. The regularity of $\infty$ in fact implies that $A_{2}$ is the sum of a bounded operator and of an operator which is similar to a self-adjoint operator in a Hilbert space (see $\S 2$ ). For the operator $A_{1}$, however, $\infty$ is in general a singular critical point if $H_{0}$ is unbounded; thus, $A_{1}$ does not generate a group of unitary operators in $\mathcal{K}_{1}$. In fact, the construction in [17] of the operator $A_{2}$ from $A_{1}$ is designed to make $\infty$ a regular critical point.

In $\S 7$, for bounded $V$, we show the existence of maximal non-negative and non-positive invariant subspaces $\mathcal{L}_{+}$and $\mathcal{L}_{-}$, respectively, for the definitizable self-adjoint operator $A_{1}$. These subspaces admit so-called angular operator representations, e.g.

$$
\mathcal{L}_{+}=\left\{\left(\begin{array}{c}
x \\
K x
\end{array}\right): x \in \mathcal{D}(K)\right\}
$$

with a closed linear operator $K$ in $\mathcal{H}$. This yields solutions on the negative and positive half-axes of the second-order initial-value problem

$$
\left(\left(\frac{\mathrm{d}}{\mathrm{d} \tau}+V\right)^{2}-H_{0}\right) v=0, \quad v(0)=v_{0}
$$

which arises from (1.2) by means of the substitution $\tau=-\mathrm{i} t, v(\tau)=u(t)$. The solutions on the positive half-axis are given by

$$
v(\tau)=\mathrm{e}^{-\tau(K+V)} v_{0}, \quad \tau \geqslant 0,
$$

and the admissible set of initial values $v_{0}$ is the domain $\mathcal{D}\left((K+V)^{2}\right)$, which in the case when $V=0$ amounts to $v_{0} \in \mathcal{D}\left(H_{0}\right)$. The solution on the positive half-axis corresponds, roughly speaking, to the spectrum of positive type and the spectrum in the upper (or lower) half-plane, whereas the solution on the negative half-axis corresponds to the spectrum of negative type and the spectrum in the upper (or lower) half-plane of $A_{1}$.

Finally, in $\S 8$, we apply the results of the previous sections to the Klein-Gordon equation (1.1) in $\mathbb{R}^{n}$. We prove that in the space $W_{2}^{-1 / 2}\left(\mathbb{R}^{n}\right)$ it has a unique classical solution if the initial values $\psi_{0}=\psi(\cdot, 0)$ and $\psi_{1}=\partial \psi(\cdot, 0) / \partial t$ belong to $W_{2}^{1}\left(\mathbb{R}^{n}\right)$ and $W_{2}^{1 / 2}\left(\mathbb{R}^{n}\right)$, respectively, and $\left(-\Delta-V^{2}\right) \psi_{0} \in W_{2}^{-1 / 2}\left(\mathbb{R}^{n}\right)$. 


\section{Preliminaries}

\subsection{Notation and definitions from spectral theory}

For Hilbert spaces $\mathcal{H}$ and $\mathcal{H}^{\prime}, L\left(\mathcal{H}, \mathcal{H}^{\prime}\right)$ denotes the space of bounded linear operators from $\mathcal{H}$ to $\mathcal{H}^{\prime}$, and we write $L(\mathcal{H})$ if $\mathcal{H}=\mathcal{H}^{\prime}$.

For a closed linear operator $A$ in a Hilbert space $\mathcal{H}$ with domain $\mathcal{D}(A)$, we denote by $\rho(A), \sigma(A)$ and $\sigma_{\mathrm{p}}(A)$ its resolvent set, spectrum and point spectrum or set of eigenvalues, respectively. For $\lambda \in \sigma_{\mathrm{p}}(A)$ the algebraic eigenspace of $A$ at $\lambda$ is denoted by $\mathcal{L}_{\lambda}(A)$. The operator $A$ is called Fredholm if its kernel is finite dimensional and its range is finite codimensional (and hence closed; see, for example, [15, Chapter IV, § 5.1]). The essential spectrum of $A$ is defined by

$$
\sigma_{\text {ess }}(A):=\{\lambda \in \mathbb{C}: A-\lambda \text { is not Fredholm }\} .
$$

An eigenvalue $\lambda_{0} \in \sigma_{\mathrm{p}}(A)$ is called of finite type if $\lambda_{0}$ is isolated (i.e. a punctured neighbourhood of $\lambda_{0}$ belongs to $\left.\rho(A)\right)$ and $A-\lambda_{0}$ is Fredholm or, equivalently, the corresponding Riesz projection is finite dimensional. The set of all eigenvalues of finite type is called the discrete spectrum of $A$ and denoted by $\sigma_{\mathrm{d}}(A)$.

For an analytic operator function $F: \mathbb{C} \rightarrow L(\mathcal{H})$, the resolvent set and the spectrum of $F$ are defined by

$$
\rho(F):=\{\lambda \in \mathbb{C}: 0 \in \rho(F(\lambda))\}, \quad \sigma(F):=\mathbb{C} \backslash \rho(F),
$$

and the point spectrum or set of eigenvalues of $F$ is the set

$$
\sigma_{\mathrm{p}}(F):=\left\{\lambda \in \mathbb{C}: 0 \in \sigma_{\mathrm{p}}(F(\lambda))\right\}
$$

(see $[\mathbf{1 4}, \mathbf{3 0}]$ ). The essential spectrum of $F$ is given by

$$
\sigma_{\text {ess }}(F):=\{\lambda \in \mathbb{C}: F(\lambda) \text { is not Fredholm }\} .
$$

An eigenvalue $\lambda_{0} \in \sigma_{\mathrm{p}}(F)$ is called of finite type if $\lambda_{0}$ is isolated (i.e. a punctured neighbourhood of $\lambda_{0}$ belongs to $\rho(F)$ ) and $F\left(\lambda_{0}\right)$ is Fredholm. If $\rho_{0} \subset \mathbb{C}$ is an open connected subset of $\mathbb{C} \backslash \sigma_{\text {ess }}(F)$ such that $\rho_{0} \cap \rho(F) \neq \emptyset$, then $\rho_{0} \cap \sigma(F)$ is at most countable with no accumulation point in $\rho_{0}$ and consists of eigenvalues of finite type of $F$, and $F(\cdot)^{-1}$ is a finitely meromorphic operator function on $\rho_{0}$. This means that $F(\cdot)^{-1}$ is a meromorphic operator function from $\rho_{0}$ to $L(\mathcal{H})$ for which the coefficients of the principal parts of the Laurent expansions at the poles of $F(\cdot)^{-1}$ are all operators of finite rank (cf. [15, Corollary XI.8.4, Theorem XVII.2.1]). Conversely, if for some open set $\rho_{0} \subset \mathbb{C}$ the operator function $F(\cdot)^{-1}$ is finitely meromorphic, then $\rho_{0} \cap \sigma_{\text {ess }}(F)=\emptyset$. The operator function $F$ is called self-adjoint if

$$
F(\bar{\lambda})=F(\lambda)^{*}, \quad \lambda \in \mathbb{C}
$$

in particular, for $\lambda \in \mathbb{R}$ the values $F(\lambda)$ are self-adjoint operators. The spectrum of a self-adjoint operator function is symmetric with respect to the real axis. 


\subsection{Self-adjoint operators in Krein spaces}

For the definition and simple properties of Krein spaces and the linear operators therein we refer the reader to $[\mathbf{4 , 7 , 2 5}$. In the following we recall some of the basic notions. A Krein space $(\mathcal{K},[\cdot, \cdot])$ is a linear space $\mathcal{K}$ which is equipped with an (indefinite) inner product $[\cdot, \cdot]$ such that $\mathcal{K}$ can be written as

$$
\mathcal{K}=\mathcal{G}_{+}[\dot{+}] \mathcal{G}_{-},
$$

where $\left(\mathcal{G}_{ \pm}, \pm[\cdot, \cdot]\right)$ are Hilbert spaces and $[\dot{+}]$ means that the sum of $\mathcal{G}_{+}$and $\mathcal{G}_{-}$is direct and $\left[\mathcal{G}_{+}, \mathcal{G}_{-}\right]=\{0\}$. The norm topology on a Krein space $\mathcal{K}$ is the norm topology of the orthogonal sum of the Hilbert spaces $\mathcal{G}_{ \pm}$in (2.1). It can be shown that this norm topology is independent of the particular decomposition (2.1); all the topological notions in $\mathcal{K}$ refer to this norm topology.

Krein spaces often arise as follows: in a given Hilbert space $(\mathcal{G},(\cdot, \cdot))$ any bounded self-adjoint operator $G$ in $\mathcal{G}$ with $0 \in \rho(G)$ induces an inner product

$$
[x, y]:=(G x, y), \quad x, y \in \mathcal{G},
$$

such that $(\mathcal{G},[\cdot, \cdot])$ becomes a Krein space. In the particular case where $G$ has the additional property $G^{2}=I$, that is, $G$ is the difference of two complementary orthogonal projections $P$ and $Q, G=P-Q$ with $P+Q=I$, one often writes $G=J$ and in the decomposition $(2.1)$ one can choose $\mathcal{G}_{+}=P \mathcal{G}, \mathcal{G}_{-}=Q \mathcal{G}$.

For a closed linear operator $A$ in a Krein space $\mathcal{K}$ with dense domain $\mathcal{D}(A)$, the (Krein space) adjoint $A^{+}$of $A$ is the densely defined operator in $\mathcal{K}$ with

$$
\mathcal{D}\left(A^{+}\right)=\{y \in \mathcal{K}:[A \cdot, y] \text { is a continuous linear functional on } \mathcal{D}(A)\}
$$

and

$$
[A x, y]=\left[x, A^{+} y\right], \quad x \in \mathcal{D}(A), y \in \mathcal{D}\left(A^{+}\right) .
$$

The operator $A$ is called symmetric in $\mathcal{K}$ if $A \subset A^{+}$, and self-adjoint if $A=A^{+}$. A selfadjoint operator $A$ in a Krein space $\mathcal{K}$ may have a non-real spectrum, which is always symmetric with respect to the real axis, and both the spectrum $\sigma(A)$ and the resolvent set $\rho(A)$ may be empty.

An element $x \in \mathcal{K}$ is called positive (respectively, non-positive, neutral, etc.) if $[x, x]>0$ (respectively, $[x, x] \leqslant 0,[x, x]=0$, etc.), a subspace of $\mathcal{K}$ is called positive (respectively, non-positive, neutral, etc.) if all its non-zero elements are positive (respectively, nonpositive, neutral, etc.). If for a self-adjoint operator $A$ in a Krein space $\mathcal{K}$ with $\lambda_{0} \in$ $\sigma_{\mathrm{p}}(A)$ all the eigenvectors at $\lambda_{0}$ are positive (respectively, negative), then $\lambda_{0}$ is called an eigenvalue of positive type (respectively, negative type). An eigenvector $x_{0}$ of $A$ at $\lambda_{0}$ that is positive or negative does not have any associated vectors.

\subsection{Definitizable operators in Krein spaces}

A self-adjoint operator $A$ in a Krein space $\mathcal{K}$ is called definitizable if $\rho(A) \neq \emptyset$ and there exists a polynomial $p$ such that

$$
[p(A) x, x] \geqslant 0, \quad x \in \mathcal{D}(p(A))
$$


The spectrum of a definitizable operator $A$ is real with the possible exception of finitely many pairs of eigenvalues $\lambda, \bar{\lambda}$, which are necessarily zeros of each definitizing polynomial $p$; at such an eigenvalue $\lambda$ or $\bar{\lambda}$ the resolvent of $A$ has a pole of order not greater than the order of $\lambda$ as a zero of the polynomial $p$. The closed linear span of all the algebraic eigenspaces $\mathcal{L}_{\lambda}(A)$ corresponding to the eigenvalues $\lambda$ of $A$ in the open upper (or lower) half-plane is a neutral subspace of $\mathcal{K}$. The algebraic eigenspaces $\mathcal{L}_{\lambda}(A), \mathcal{L}_{\bar{\lambda}}(A)$ corresponding to non-real $\lambda, \bar{\lambda} \in \sigma_{\mathrm{p}}(A)$ are skewly linked, that is, to each non-zero $x \in \mathcal{L}_{\lambda}(A)$ there exists a $y \in \mathcal{L}_{\bar{\lambda}}(A)$ such that $[x, y] \neq 0$ and to each non-zero $y \in \mathcal{L}_{\bar{\lambda}}(A)$ there exists an $x \in \mathcal{L}_{\lambda}(A)$ such that $[x, y] \neq 0$.

If $\lambda \in \sigma_{\mathrm{p}}(A)$, the maximal dimension (up to $\infty$ ) of a non-negative (non-positive, respectively) subspace of $\mathcal{L}_{\lambda}(A)$ is denoted by $\kappa_{\lambda}^{+}(A)\left(\kappa_{\lambda}^{-}(A)\right.$, respectively), and the dimension of the so-called isotropic subspace $\mathcal{L}_{\lambda}(A) \cap \mathcal{L}_{\lambda}(A)^{[\perp]}$ of $\mathcal{L}_{\lambda}(A)$ is denoted by $\kappa_{\lambda}^{\mathrm{o}}(A)$. Observe that if, for example, $\mathcal{L}_{\lambda}(A)$ is one-dimensional and neutral, then $\kappa_{\lambda}^{+}(A)=\kappa_{\lambda}^{-}(A)=\kappa_{\lambda}^{\mathrm{o}}(A)=1$; for a non-real eigenvalue $\lambda$ of $A$ the number $\kappa_{\lambda}^{\mathrm{o}}(A)$ coincides with the dimension of $\mathcal{L}_{\lambda}(A)$.

A definitizable operator $A$ with definitizing polynomial $p$ has a spectral function with critical points. To introduce it, we call a bounded real interval $\Gamma$ admissible for the operator $A$ if some definitizing polynomial $p$ of $A$ does not vanish at the end points of $\Gamma$. Then, for each admissible bounded interval $\Gamma$, there exists an orthogonal projection $E(\Gamma)$ in $\mathcal{K}$ such that the range $E(\Gamma) \mathcal{K}$ is invariant under $A$, the spectrum of the restriction $\left.A\right|_{E(\Gamma) \mathcal{K}}$ is contained in $\bar{\Gamma}$, and the real spectrum of the restriction $\left.A\right|_{(I-E(\Gamma)) \mathcal{K}}$ is contained in $\overline{\mathbb{R} \backslash \Gamma}$.

A real spectral point $\lambda \in \sigma(A)$ is called of positive type if there exists an admissible open interval $\Gamma$ such that $\lambda \in \Gamma$ and $(E(\Gamma) \mathcal{K},[\cdot, \cdot])$ is a Hilbert space; this is equivalent to the fact that $[x, x] \geqslant 0, x \in E(\Gamma) \mathcal{K}$ (which implies that $[x, x]>0$ if $x \neq 0$ ). The set of all spectral points of positive type of $A$ is denoted by $\sigma_{+}(A)$. Clearly, if $(E(\Gamma) \mathcal{K},[\cdot, \cdot])$ is a Hilbert space, the restriction $\left.A\right|_{E(\Gamma) \mathcal{K}}$ has the same spectral properties as a self-adjoint operator in a Hilbert space. If a definitizing polynomial $p$ is positive on an admissible interval $\Gamma$, then $\Gamma \cap \sigma(A)$ consists only of spectral points of positive type. Similarly, a real point $\lambda \in \sigma(A)$ for which there exists an open admissible interval $\Gamma$ such that $\lambda \in \Gamma$ and $(E(\Gamma) \mathcal{K},-[\cdot, \cdot])$ is a Hilbert space is called a spectral point of negative type of $A$; the set of all spectral points of negative type of $A$ is denoted by $\sigma_{-}(A)$. Finally, $\lambda_{0} \in \mathbb{R}$ is called a critical point of $A$ if, for each admissible open interval $\Gamma$ with $\lambda_{0} \in \Gamma$, the range $E(\Gamma) \mathcal{K}$ contains both positive and negative elements. The set of all critical points of $A$ is denoted by $\sigma_{\text {crit }}(A)$; it is always finite. In fact, each critical point is a zero of every definitizing polynomial $p$. From these definitions it follows that

$$
\sigma(A) \cap \mathbb{R}=\sigma_{+}(A) \cup \sigma_{-}(A) \cup \sigma_{\text {crit }}(A) .
$$

A real eigenvalue of $A$ with a neutral eigenvector is always a critical point of $A$. An eigenvalue $\lambda$ of positive type is a critical point of $A$ if in each neighbourhood of $\lambda$ there are spectral points of negative type of $A$. If, however, the eigenvalue $\lambda$ of positive type is an isolated spectral point, then it is a spectral point of positive type.

Similarly, $\infty$ is called a critical point of $A$ if, outside of each compact real interval, there are spectral points of positive and of negative type of $A$. If $\infty$ is a critical point 
of the self-adjoint operator $A$, it is called a regular critical point of $A$ if there exists a constant $\gamma>0$ such that $\|E(\Gamma)\| \leqslant \gamma$ for all sufficiently large intervals $\Gamma$ centred at 0 ; otherwise, $\infty$ is called a singular critical point; here $\|\cdot\|$ denotes the operator norm corresponding to the norm in $\mathcal{K}$ induced by any of the equivalent decompositions (2.1). Note that the norm of a self-adjoint projection in a Krein space can be arbitrarily large. If $\infty$ is a regular critical point of $A$, then outside of a sufficiently large compact interval the operator $A$ has the same spectral properties as a self-adjoint operator in a Hilbert space; in particular, the bounded operators $\mathrm{e}^{\mathrm{i} t A}, t \in \mathbb{R}$, can be defined and form a group of unitary operators in $\mathcal{K}$. Recall that a unitary operator $U$ in a Krein space $\mathcal{K}$ is a bounded operator such that

$$
U U^{+}=U^{+} U=I
$$

The operators occurring in this paper belong to a special class of definitizable operators: they are self-adjoint operators $A$ in a Krein space $\mathcal{K}$ for which $\rho(A) \neq 0$ and the sesquilinear form

$$
[A x, y], \quad x, y \in \mathcal{D}(A)
$$

has a finite number $\kappa$ of negative squares; recall that the latter means that each subspace $\mathcal{L}$ of $\mathcal{K}$ for which

$$
[A x, x]<0, \quad x \in \mathcal{L}, x \neq 0,
$$

is of dimension less than or equal to $\kappa$ and for at least one $\kappa$-dimensional subspace $\mathcal{L}$ the relation $(2.3)$ holds. In this case the definitizing polynomial is of the form $p(\lambda)=\lambda q(\lambda) \overline{q(\bar{\lambda})}$ with some polynomial $q$ of degree less than or equal to $\kappa$. Then all the algebraic eigenspaces corresponding to non-real eigenvalues are finite dimensional, the positive spectrum of $A$ consists of spectral points of positive type with the possible exception of a finite number of eigenvalues with a negative or neutral eigenvector, and the negative spectrum of $A$ consists of spectral points of negative type with the possible exception of a finite number of eigenvalues with a positive or neutral eigenvector. If, additionally, $A$ is boundedly invertible, the following equality holds:

$$
\kappa=\sum_{\lambda \in \sigma_{\mathrm{p}}(A) \cap(0,+\infty)} \kappa_{\lambda}^{-}(A)+\sum_{\lambda \in \sigma_{\mathrm{p}}(A) \cap(-\infty, 0)} \kappa_{\lambda}^{+}(A)+\sum_{\lambda \in \sigma_{\mathrm{p}}(A) \cap \mathbb{C}^{+}} \kappa_{\lambda}^{\mathrm{o}}(A) .
$$

The Krein space $\mathcal{K}$ is called a Pontryagin space with negative index $\kappa$ if in one (and hence in all) decompositions of the form (2.1) the space $\mathcal{G}_{-}$has finite dimension $\kappa$. Any self-adjoint operator $A$ in a Pontryagin space is definitizable and hence has a spectral function with critical points. With the exception of finitely many points, the real spectral points of $A$ are of positive type; the exceptional points are eigenvalues with a negative or neutral eigenvector and

$$
\kappa=\sum_{\lambda \in \sigma_{p}(A) \cap \mathbb{R}} \kappa_{\lambda}^{-}(A)+\sum_{\lambda \in \sigma_{p}(A) \cap \mathbb{C}^{+}} \kappa_{\lambda}^{\circ}(A) .
$$




\section{Operators associated with the abstract Klein-Gordon equation: $A_{1}$ in $\mathcal{H} \oplus \mathcal{H}$}

Let $(\mathcal{H},(\cdot, \cdot))$ be a Hilbert space with corresponding norm $\|\cdot\|$, let $H_{0}$ be a uniformly positive self-adjoint operator in $\mathcal{H}, H_{0} \geqslant m^{2}>0$, and let $V$ be a densely defined symmetric operator in $\mathcal{H}$.

By $\mathcal{G}_{1}$ we denote the orthogonal sum $\mathcal{G}_{1}:=\mathcal{H} \oplus \mathcal{H}$ with its inner product

$$
\left(\boldsymbol{x}, \boldsymbol{x}^{\prime}\right)_{\mathcal{G}_{1}}:=\left(x, x^{\prime}\right)+\left(y, y^{\prime}\right), \quad \boldsymbol{x}=(x y)^{\mathrm{T}}, \boldsymbol{x}^{\prime}=\left(x^{\prime} y^{\prime}\right)^{\mathrm{T}} \in \mathcal{G}_{1} .
$$

Equipped with the indefinite inner product

$$
\left[\boldsymbol{x}, \boldsymbol{x}^{\prime}\right]:=\left(x, y^{\prime}\right)+\left(y, x^{\prime}\right), \quad \boldsymbol{x}=(x y)^{\mathrm{T}}, \boldsymbol{x}^{\prime}=\left(x^{\prime} y^{\prime}\right)^{\mathrm{T}} \in \mathcal{G}_{1},
$$

the space $\mathcal{K}_{1}:=\left(\mathcal{G}_{1},[\cdot, \cdot]\right)$ becomes a Krein space. This is clear since the inner product $[\cdot, \cdot]$ can be defined by $\left[\boldsymbol{x}, \boldsymbol{x}^{\prime}\right]=\left(G \boldsymbol{x}, \boldsymbol{x}^{\prime}\right)_{\mathcal{G}_{1}}$ with the Gram operator

$$
G=\left(\begin{array}{ll}
0 & I \\
I & 0
\end{array}\right) .
$$

In $\mathcal{G}_{1}=\mathcal{H} \oplus \mathcal{H}$, with the abstract first-order differential equation (1.7), we associate the block operator matrix $\hat{A}_{1}$ given by

$$
\hat{A}_{1}:=\left(\begin{array}{cc}
V & I \\
H_{0} & V
\end{array}\right) .
$$

With its natural domain $\mathcal{D}\left(\hat{A}_{1}\right):=\left(\mathcal{D}(V) \cap \mathcal{D}\left(H_{0}\right)\right) \oplus \mathcal{D}(V)$, the operator $\hat{A}_{1}$ need not be densely defined nor closable. The main assumption here and below is as follows.

Assumption 3.1. $\mathcal{D}\left(H_{0}^{1 / 2}\right) \subset \mathcal{D}(V)$.

Since $V$ is closable (with closure denoted by $\bar{V}$ ), Assumption 3.1 is satisfied if and only if $V$ is $H_{0}^{1 / 2}$-bounded (see [22, $\S \S$ IV.1.1, IV.1.3]). Since, in addition, $H_{0}$ is assumed to be boundedly invertible, Assumption 3.1 implies that the operator

$$
S:=V H_{0}^{-1 / 2}
$$

is defined on all of $\mathcal{H}$ and bounded in $\mathcal{H}$. Together with

$$
H_{0}^{-1 / 2} V \subset H_{0}^{-1 / 2} V^{*} \subset\left(V H_{0}^{-1 / 2}\right)^{*}=S^{*},
$$

this shows that $\overline{H_{0}^{-1 / 2} V}=S^{*}$.

Under Assumption 3.1, the domain of $\hat{A}_{1}$ takes the form

$$
\mathcal{D}\left(\hat{A}_{1}\right)=\left\{\left(\begin{array}{l}
x \\
y
\end{array}\right) \in \mathcal{H} \oplus \mathcal{H}: x \in \mathcal{D}\left(H_{0}\right), y \in \mathcal{D}(V)\right\} .
$$


Theorem 3.2. If $\mathcal{D}\left(H_{0}^{1 / 2}\right) \subset \mathcal{D}(V)$, then $\hat{A}_{1}$ is essentially self-adjoint in the Krein space $\mathcal{K}_{1}$. Its closure is the self-adjoint operator $A_{1}=\left(\hat{A}_{1}\right)^{+}$given by

$$
\begin{aligned}
\mathcal{D}\left(A_{1}\right) & =\left\{\left(\begin{array}{l}
x \\
y
\end{array}\right) \in \mathcal{H} \oplus \mathcal{H}: x \in \mathcal{D}\left(H_{0}^{1 / 2}\right), H_{0}^{1 / 2} x+S^{*} y \in \mathcal{D}\left(H_{0}^{1 / 2}\right)\right\}, \\
A_{1}\left(\begin{array}{l}
x \\
y
\end{array}\right) & =\left(\begin{array}{c}
V x+y \\
H_{0}^{1 / 2}\left(H_{0}^{1 / 2} x+S^{*} y\right)
\end{array}\right) .
\end{aligned}
$$

Proof. First we show that

$$
\left(\hat{A}_{1}\right)^{+}=A_{1} .
$$

In order to prove the inclusion $\left(\hat{A}_{1}\right)^{+} \subset A_{1}$, let $\boldsymbol{x}=(x y)^{\mathrm{T}} \in \mathcal{D}\left(\left(\hat{A}_{1}\right)^{+}\right)$. Then, for $\left(\hat{A}_{1}\right)^{+} \boldsymbol{x}=: \boldsymbol{u}=(u v)^{\mathrm{T}} \in \mathcal{G}_{1}=\mathcal{H} \oplus \mathcal{H}$, we have

$$
\left[\hat{A}_{1} \boldsymbol{x}^{\prime}, \boldsymbol{x}\right]=\left[\boldsymbol{x}^{\prime}, \boldsymbol{u}\right], \quad \boldsymbol{x}^{\prime}=\left(x^{\prime} y^{\prime}\right)^{\mathrm{T}} \in \mathcal{D}\left(\hat{A}_{1}\right),
$$

that is,

$$
\left(V x^{\prime}+y^{\prime}, y\right)+\left(H_{0} x^{\prime}+V y^{\prime}, x\right)=\left(x^{\prime}, v\right)+\left(y^{\prime}, u\right), \quad x^{\prime} \in \mathcal{D}\left(H_{0}\right), y^{\prime} \in \mathcal{D}(V) .
$$

Choosing $y^{\prime}=0$, we conclude that for all $x^{\prime} \in \mathcal{D}\left(H_{0}\right)$ we have the equality

$$
\begin{aligned}
\left(V x^{\prime}, y\right)+\left(H_{0} x^{\prime}, x\right)= & \left(x^{\prime}, v\right) \\
& \Longleftrightarrow\left(V H_{0}^{-1 / 2}\left(H_{0}^{1 / 2} x^{\prime}\right), y\right)+\left(H_{0}^{1 / 2}\left(H_{0}^{1 / 2} x^{\prime}\right), x\right)=\left(H_{0}^{-1 / 2}\left(H_{0}^{1 / 2} x^{\prime}\right), v\right) \\
& \Longleftrightarrow\left(H_{0}^{1 / 2} x^{\prime}, S^{*} y\right)+\left(H_{0}^{1 / 2}\left(H_{0}^{1 / 2} x^{\prime}\right), x\right)=\left(H_{0}^{1 / 2} x^{\prime}, H_{0}^{-1 / 2} v\right) \\
& \Longleftrightarrow\left(H_{0}^{1 / 2}\left(H_{0}^{1 / 2} x^{\prime}\right), x\right)=\left(H_{0}^{1 / 2} x^{\prime}, H_{0}^{-1 / 2} v-S^{*} y\right) \\
& \Longleftrightarrow\left(H_{0}^{1 / 2} w^{\prime}, x\right)=\left(w^{\prime}, H_{0}^{-1 / 2} v-S^{*} y\right)
\end{aligned}
$$

with $w^{\prime}:=H_{0}^{1 / 2} x^{\prime}$ being an arbitrary element of $\mathcal{D}\left(H_{0}^{1 / 2}\right)$. Hence, $x \in \mathcal{D}\left(H_{0}^{1 / 2}\right)$ and

$$
H_{0}^{1 / 2} x=H_{0}^{-1 / 2} v-S^{*} y
$$

that is,

$$
H_{0}^{1 / 2} x+S^{*} y=H_{0}^{-1 / 2} v \in \mathcal{D}\left(H_{0}^{1 / 2}\right)
$$

and

$$
v=H_{0}^{1 / 2}\left(H_{0}^{1 / 2} x+S^{*} y\right) .
$$

Choosing $x^{\prime}=0$ in (3.7) and using the symmetry of $V$, we obtain that, for all $y^{\prime} \in \mathcal{D}(V)$,

$$
\left(y^{\prime}, y\right)+\left(V y^{\prime}, x\right)=\left(y^{\prime}, u\right)
$$

Since $x \in \mathcal{D}\left(H_{0}^{1 / 2}\right) \subset \mathcal{D}(V)$ and $\mathcal{D}(V)$ is dense in $\mathcal{H}$, we see that $u=V x+y$. 
The inclusion $A_{1} \subset\left(\hat{A}_{1}\right)^{+}$follows if we observe that, for arbitrary $\boldsymbol{x}=\left(\begin{array}{ll}x y & y\end{array}\right)^{\mathrm{T}} \in \mathcal{D}\left(A_{1}\right)$ and $\boldsymbol{x}^{\prime}=\left(x^{\prime} y^{\prime}\right)^{\mathrm{T}} \in \mathcal{D}\left(\hat{A}_{1}\right)$,

$$
\begin{aligned}
{\left[\hat{A}_{1} \boldsymbol{x}^{\prime}, \boldsymbol{x}\right] } & =\left(V x^{\prime}, y\right)+\left(y^{\prime}, y\right)+\left(H_{0} x^{\prime}, x\right)+\left(V y^{\prime}, x\right) \\
& =\left(H_{0}^{1 / 2} x^{\prime}, S^{*} y\right)+\left(y^{\prime}, y\right)+\left(H_{0}^{1 / 2} x^{\prime}, H_{0}^{1 / 2} x\right)+\left(V y^{\prime}, x\right) \\
& =\left(H_{0}^{1 / 2} x^{\prime}, H_{0}^{1 / 2} x+S^{*} y\right)+\left(y^{\prime}, V x+y\right) \\
& =\left[\boldsymbol{x}^{\prime}, A_{1} \boldsymbol{x}\right] .
\end{aligned}
$$

By the definition of $\hat{A}_{1}$ and $A_{1}$ and by (3.6), we have $\hat{A}_{1} \subset A_{1}=\left(\hat{A}_{1}\right)^{+}$. Thus, $\hat{A}_{1}$ is symmetric in $\mathcal{K}_{1}$ and hence closable. Since $\left(\hat{A}_{1}\right)^{+}$is closed, it follows that

$$
\overline{\hat{A}_{1}} \subset\left(\hat{A}_{1}\right)^{+}=A_{1},
$$

and the theorem is proved if we show that $A_{1} \subset \overline{\hat{A}_{1}}$.

To this end, let $(x y)^{\mathrm{T}} \in \mathcal{D}\left(A_{1}\right)$. Then $x \in \mathcal{D}\left(H_{0}^{1 / 2}\right)$ and $y \in \mathcal{H}$ are such that $z:=$ $H_{0}^{1 / 2} x+S^{*} y \in \mathcal{D}\left(H_{0}^{1 / 2}\right)$. Since $\mathcal{D}(V)$ is dense in $\mathcal{H}$, there exists a sequence $\left(y_{n}\right) \subset \mathcal{D}(V)$ with $y_{n} \rightarrow y$ and, since $S$ is bounded, $H_{0}^{-1 / 2} V y_{n}=S^{*} y_{n} \rightarrow S^{*} y$. If we define

$$
\tilde{x}_{n}:=z-H_{0}^{-1 / 2} V y_{n} \in \mathcal{D}\left(H_{0}^{1 / 2}\right) \quad \text { and } \quad x_{n}:=H_{0}^{-1 / 2} \tilde{x}_{n} \in \mathcal{D}\left(H_{0}\right),
$$

then we have, for $n \rightarrow \infty$,

$$
\begin{gathered}
x_{n}=H_{0}^{-1 / 2} z-H_{0}^{-1 / 2} H_{0}^{-1 / 2} V y_{n} \rightarrow H_{0}^{-1 / 2}\left(H_{0}^{1 / 2} x+S^{*} y\right)-H_{0}^{-1 / 2} S^{*} y=x, \\
H_{0}^{1 / 2} x_{n}=\tilde{x}_{n} \rightarrow z-S^{*} y=H_{0}^{1 / 2} x .
\end{gathered}
$$

Since $V$ is $H_{0}^{1 / 2}$-bounded by Assumption 3.1, this implies that also $\left(V x_{n}\right)$ and hence $\left(V x_{n}+y_{n}\right)$ converges. Finally,

$$
H_{0} x_{n}+V y_{n}=H_{0}^{1 / 2}\left(H_{0}^{1 / 2} x_{n}+H_{0}^{-1 / 2} V y_{n}\right)=H_{0}^{1 / 2}\left(\tilde{x}_{n}+H_{0}^{-1 / 2} V y_{n}\right)=H_{0}^{1 / 2} z,
$$

and hence $\left(H_{0} x_{n}+V y_{n}\right)$ converges. This proves that $(x y)^{\mathrm{T}} \in \mathcal{D}\left(\overline{\hat{A}_{1}}\right)$.

In order to ensure that the resolvent set of $A_{1}$ is non-empty, an additional condition is required in $\S 5$. In this respect, the self-adjoint quadratic operator polynomial

$$
L_{1}(\lambda):=I-\left(S-\lambda H_{0}^{-1 / 2}\right)\left(S^{*}-\lambda H_{0}^{-1 / 2}\right), \quad \lambda \in \mathbb{C},
$$

in the Hilbert space $\mathcal{H}$ is useful as it reflects the spectral properties of $A_{1}$; note that, according to Assumption 3.1, the values $L_{1}(\lambda)$ are bounded operators in $\mathcal{H}$.

Proposition 3.3. If $\mathcal{D}\left(H_{0}^{1 / 2}\right) \subset \mathcal{D}(V)$, then, for $\lambda \in \mathbb{C}$,

$$
A_{1}-\lambda=\left(\begin{array}{cc}
I & \left(S-\lambda H_{0}^{-1 / 2}\right) H_{0}^{1 / 2} \\
0 & H_{0}
\end{array}\right)\left(\begin{array}{cc}
0 & L_{1}(\lambda) \\
I & H_{0}^{-1 / 2}\left(S^{*}-\lambda H_{0}^{-1 / 2}\right)
\end{array}\right) .
$$


Proof. The formal equality in (3.9) follows immediately from formula (3.8). To prove the equality of the domains, we observe that $\left(S-\lambda H_{0}^{-1 / 2}\right) H_{0}^{1 / 2}=V-\lambda$ on $\mathcal{D}\left(H_{0}\right)$. Then the domain $\mathcal{D}_{1}$ of the product on the right-hand side of (3.9) is given by

$$
\begin{aligned}
\mathcal{D}_{1} & =\left\{\left(\begin{array}{l}
x \\
y
\end{array}\right) \in \mathcal{H} \oplus \mathcal{H}: x+H_{0}^{-1 / 2}\left(S^{*}-\lambda H_{0}^{-1 / 2}\right) y \in \mathcal{D}\left(H_{0}\right)\right\} \\
& =\left\{\left(\begin{array}{l}
x \\
y
\end{array}\right) \in \mathcal{H} \oplus \mathcal{H}: x+H_{0}^{-1 / 2} S^{*} y \in \mathcal{D}\left(H_{0}\right)\right\} \\
& =\left\{\left(\begin{array}{l}
x \\
y
\end{array}\right) \in \mathcal{H} \oplus \mathcal{H}: x \in \mathcal{D}\left(H_{0}^{1 / 2}\right), H_{0}^{1 / 2} x+S^{*} y \in \mathcal{D}\left(H_{0}^{1 / 2}\right)\right\},
\end{aligned}
$$

which coincides with the domain of $A_{1}$ by Theorem 3.2 .

Proposition 3.4. Let $\mathcal{D}\left(H_{0}^{1 / 2}\right) \subset \mathcal{D}(V)$. Then $\rho\left(L_{1}\right) \subset \rho\left(A_{1}\right)$ and, for $\lambda \in \rho\left(L_{1}\right)$,

$$
\begin{aligned}
\left(A_{1}\right. & -\lambda)^{-1} \\
& =\left(\begin{array}{cc}
-H_{0}^{-1 / 2}\left(S^{*}-\lambda H_{0}^{-1 / 2}\right) L_{1}(\lambda)^{-1} & I \\
L_{1}(\lambda)^{-1} & 0
\end{array}\right)\left(\begin{array}{cc}
I & -\left(S-\lambda H_{0}^{-1 / 2}\right) H_{0}^{-1 / 2} \\
0 & H_{0}^{-1}
\end{array}\right) \\
& =\left(\begin{array}{cc}
0 & H_{0}^{-1} \\
0 & 0
\end{array}\right)+\left(\begin{array}{cc}
-H_{0}^{-1 / 2}\left(S^{*}-\lambda H_{0}^{-1 / 2}\right) \\
I
\end{array}\right) L_{1}(\lambda)^{-1}\left(\begin{array}{ll}
I & -\left(S-\lambda H_{0}^{-1 / 2}\right) H_{0}^{-1 / 2}
\end{array}\right) .
\end{aligned}
$$

Moreover,

$$
\sigma_{\text {ess }}\left(A_{1}\right) \subset \sigma_{\text {ess }}\left(L_{1}\right)
$$

Proof. The first and the second claim are immediate consequences of the factorization (3.9). If $\lambda_{0} \notin \sigma_{\text {ess }}\left(L_{1}\right)$, then $L_{1}(\cdot)^{-1}$ is a finitely meromorphic operator function in a neighbourhood of $\lambda_{0}$ and hence so is $\left(A_{1}-\cdot\right)^{-1}$ by the second formula for $\left(A_{1}-\lambda\right)^{-1}$ above. This shows that $\lambda_{0} \notin \sigma_{\text {ess }}\left(A_{1}\right)$.

\section{Operators associated with the abstract Klein-Gordon equation: \\ $A_{2}$ in $\mathcal{H}_{1 / 4} \oplus \mathcal{H}_{-1 / 4}$}

In order to associate a second operator $A_{2}$ with the abstract Klein-Gordon equation (1.2), we introduce a scale of Hilbert spaces $\left(\mathcal{H}_{\alpha},\|\cdot\|_{\alpha}\right),-1 \leqslant \alpha \leqslant 1$, induced by the operator $H_{0}$ as follows. If $0 \leqslant \alpha \leqslant 1$, we set

$$
\mathcal{H}_{\alpha}:=\mathcal{D}\left(H_{0}^{\alpha}\right), \quad\|x\|_{\alpha}:=\left\|H_{0}^{\alpha} x\right\|, \quad x \in \mathcal{H}_{\alpha}, 0 \leqslant \alpha \leqslant 1 .
$$

Obviously, $\mathcal{H}_{0}=\mathcal{H}$. If $-1 \leqslant \alpha<0$, then $\mathcal{H}_{\alpha}$ is defined to be the corresponding space with negative norm (see $[6]$ ), which can also be considered as the completion of $\mathcal{D}\left(H_{0}^{\alpha}\right)=\mathcal{H}$ with respect to the norm

$$
\|x\|_{\alpha}:=\left\|H_{0}^{\alpha} x\right\|, \quad x \in \mathcal{H},-1 \leqslant \alpha<0 .
$$


All powers of $H_{0}$ extend in a natural way to operators between the spaces of the scale $\mathcal{H}_{\alpha},-1 \leqslant \alpha \leqslant 1$; in particular, $H_{0}^{\beta} \in L\left(\mathcal{H}_{\alpha}, \mathcal{H}_{\alpha-\beta}\right)$ for $\alpha, \beta, \alpha-\beta \in[-1,1]$. The duality between $\mathcal{H}_{\alpha}$ and $\mathcal{H}_{-\alpha}$ is again denoted by $(\cdot, \cdot)$ :

$$
(x, y):=\left(H_{0}^{\alpha} x, H_{0}^{-\alpha} y\right), \quad x \in \mathcal{H}_{\alpha}, y \in \mathcal{H}_{-\alpha} .
$$

Let $\mathcal{G}_{2}$ be the orthogonal sum $\mathcal{G}_{2}:=\mathcal{H}_{1 / 4} \oplus \mathcal{H}_{-1 / 4}$ with its inner product

$$
\left(\boldsymbol{x}, \boldsymbol{x}^{\prime}\right)_{\mathcal{G}_{2}}=\left(H_{0}^{1 / 4} x, H_{0}^{1 / 4} x^{\prime}\right)+\left(H_{0}^{-1 / 4} y, H_{0}^{-1 / 4} y^{\prime}\right)
$$

for $\boldsymbol{x}=\left(\begin{array}{ll}x y & y\end{array}\right)^{\mathrm{T}}, \boldsymbol{x}^{\prime}=\left(x^{\prime} y^{\prime}\right)^{\mathrm{T}} \in \mathcal{G}_{2}$. In addition, we equip the space $\mathcal{G}_{2}$ with the indefinite inner product

$$
\left[\boldsymbol{x}, \boldsymbol{x}^{\prime}\right]:=\left(H_{0}^{1 / 4} x, H_{0}^{-1 / 4} y^{\prime}\right)+\left(H_{0}^{-1 / 4} y, H_{0}^{1 / 4} x^{\prime}\right)
$$

for $\boldsymbol{x}=\left(\begin{array}{ll}x y & { }^{\mathrm{T}}, \boldsymbol{x}^{\prime}=\left(x^{\prime} y^{\prime}\right.\end{array}\right)^{\mathrm{T}} \in \mathcal{G}_{2}$, that is,

$$
\left[\boldsymbol{x}, \boldsymbol{x}^{\prime}\right]=\left(x, y^{\prime}\right)+\left(y, x^{\prime}\right)
$$

the brackets on the right-hand side of (4.3) denote the duality (4.2) between $\mathcal{H}_{1 / 4}$ and $\mathcal{H}_{-1 / 4}$ and vice versa.

The space $\mathcal{K}_{2}:=\left(\mathcal{G}_{2},[\cdot, \cdot]\right)$ is a Krein space. To see this, we observe that $H_{0}^{-1 / 2}$ is a unitary mapping from $\mathcal{G}_{-1 / 4}$ onto $\mathcal{G}_{1 / 4}, H_{0}^{1 / 2}$ is a unitary mapping from $\mathcal{G}_{1 / 4}$ onto $\mathcal{G}_{-1 / 4}$, and that

$$
\left[\boldsymbol{x}, \boldsymbol{x}^{\prime}\right]=\left(y, x^{\prime}\right)+\left(x, y^{\prime}\right)=\left(\left(\begin{array}{c}
H_{0}^{-1 / 2} y \\
H_{0}^{1 / 2} x
\end{array}\right),\left(\begin{array}{c}
x^{\prime} \\
y^{\prime}
\end{array}\right)\right)_{\mathcal{G}_{2}}=\left(J \boldsymbol{x}, \boldsymbol{x}^{\prime}\right)_{\mathcal{G}_{2}} ;
$$

here the operator $J$ in $\mathcal{G}_{2}=\mathcal{H}_{1 / 4} \oplus \mathcal{H}_{-1 / 4}$ is given by

$$
J:=\left(\begin{array}{cc}
0 & H_{0}^{-1 / 2} \\
H_{0}^{1 / 2} & 0
\end{array}\right)
$$

and satisfies $J=J^{*}$ as well as $J^{2}=I$ ( see $\left.\S 2.2\right)$.

Imposing Assumption 3.1, that is, $\mathcal{D}\left(H_{0}^{1 / 2}\right) \subset \mathcal{D}(V)$, we may consider the symmetric operator $V$ also as an operator in the scale of spaces $\mathcal{H}_{\alpha},-1 \leqslant \alpha \leqslant 1$.

Remark 4.1. Assumption 3.1 is equivalent to the boundedness of $V$ regarded as an operator from $\mathcal{H}_{1 / 2}$ to $\mathcal{H}$, that is, $V \in L\left(\mathcal{H}_{1 / 2}, \mathcal{H}\right)$. Due to its symmetry, $V$ then admits an extension as a bounded operator from $\mathcal{H}$ to $\mathcal{H}_{-1 / 2}$; by interpolation, it can also be defined as a bounded operator from $\mathcal{H}_{\alpha}$ into $\mathcal{H}_{\alpha-1 / 2}$ for all $\alpha \in\left[0, \frac{1}{2}\right]$, see [39, Chapter IX.4, Appendix, Example 3]. All these extensions and restrictions of the operator $V$, originally given in $\mathcal{H}$, which act between the spaces of the scale $\mathcal{H}_{\alpha}$, are also denoted by $V$ :

$$
V \in L\left(\mathcal{H}_{\alpha}, \mathcal{H}_{\alpha-1 / 2}\right), \quad \alpha \in\left[0, \frac{1}{2}\right] .
$$

As a consequence of (4.5), for the corresponding extensions and restrictions of the operators $S=V H_{0}^{-1 / 2}$ and the adjoint $S^{*}=\left(V H_{0}^{-1 / 2}\right)^{*}$ of $S$ in $\mathcal{H}$, we have

$$
S=V H_{0}^{-1 / 2} \in L\left(\mathcal{H}_{\alpha}\right), \quad \alpha \in\left[-\frac{1}{2}, 0\right]
$$


and

$$
S^{*}=H_{0}^{-1 / 2} V \in L\left(\mathcal{H}_{\alpha}\right), \quad \alpha \in\left[0, \frac{1}{2}\right] .
$$

Note that in $\S 3$ the operator $S^{*}$ had to be written as $S^{*}=\overline{H_{0}^{1 / 2} V}$ since there $V$ acts only within $\mathcal{H}$ and thus requires the domain $\mathcal{D}(V) \subset \mathcal{H}$; observe also that in $\mathcal{H}_{\alpha}, \alpha \neq 0$, the operator $S^{*}$ is not the adjoint of $S$.

Under Assumption 3.1, we now consider the operator $A_{2}$ in $\mathcal{G}_{2}$ given by

$$
\begin{aligned}
\mathcal{D}\left(A_{2}\right) & :=\left\{\left(\begin{array}{l}
x \\
y
\end{array}\right) \in \mathcal{H}_{1 / 4} \oplus \mathcal{H}_{-1 / 4}: x \in \mathcal{D}\left(H_{0}^{1 / 2}\right), y \in \mathcal{H},\right. \\
\left.V x+y \in \mathcal{H}_{1 / 4}, H_{0} x+V y \in \mathcal{H}_{-1 / 4}\right\} & \\
A_{2}\left(\begin{array}{l}
x \\
y
\end{array}\right) & :=\left(\begin{array}{c}
V x+y \\
H_{0} x+V y
\end{array}\right) .
\end{aligned}
$$

Remark 4.2. The domain of $A_{2}$ can also be written as

$$
\mathcal{D}\left(A_{2}\right)=\left\{\left(\begin{array}{l}
x \\
y
\end{array}\right) \in \mathcal{H}_{1 / 4} \oplus \mathcal{H}_{-1 / 4}: y \in \mathcal{H}, V x+y \in \mathcal{H}_{1 / 4}, H_{0} x+V y \in \mathcal{H}_{-1 / 4}\right\} ;
$$

in fact, if $y \in \mathcal{H}$, then $H_{0} x+V y \in \mathcal{H}_{-1 / 4}$ automatically implies $x \in \mathcal{D}\left(H_{0}^{1 / 2}\right)$.

In the following, we first show that $A_{2}$ is a symmetric operator in the Krein space $\mathcal{K}_{2}$. In the theorem below we give a sufficient condition for the self-adjointness of $A_{2}$ in $\mathcal{K}_{2}$.

Proposition 4.3. If $\mathcal{D}\left(H_{0}^{1 / 2}\right) \subset \mathcal{D}(V)$, then $A_{2}$ is symmetric in $\mathcal{K}_{2}$.

Proof. Let $\boldsymbol{x}=(x y)^{\mathrm{T}} \in \mathcal{D}\left(A_{2}\right) \subset \mathcal{D}\left(H_{0}^{1 / 2}\right) \oplus \mathcal{H}$. Then, according to the formula (4.3) for the inner product $[\cdot, \cdot]$,

$$
\left[A_{2} \boldsymbol{x}, \boldsymbol{x}\right]=(V x+y, y)+\left(H_{0} x+V y, x\right)=(V x, y)+(y, y)+\left(H_{0} x, x\right)+(V y, x) .
$$

Note that the first two terms are the inner products in $\mathcal{H}$, whereas the last two terms denote the duality between $\mathcal{G}_{-1 / 2}$ and $\mathcal{G}_{1 / 2}$. Since

$$
(V y, x)=\left(H_{0}^{-1 / 2} V y, H_{0}^{1 / 2} x\right)=\left(S^{*} y, H_{0}^{1 / 2} x\right)=\left(y, S H_{0}^{1 / 2} x\right)=(y, V x)=\overline{(V x, y)},
$$

it follows that $\left[A_{2} \boldsymbol{x}, \boldsymbol{x}\right] \in \mathbb{R}$.

Theorem 4.4. Suppose that $\mathcal{D}\left(H_{0}^{1 / 2}\right) \subset \mathcal{D}(V)$. Then

$$
\rho\left(L_{1}\right) \subset \rho\left(A_{2}\right),
$$

the operator $A_{2}$ is self-adjoint in $\mathcal{K}_{2}$ if $\rho\left(L_{1}\right) \neq \emptyset$ and, for $\lambda \in \rho\left(L_{1}\right)$,

$$
\begin{aligned}
\left(A_{2}-\lambda\right)^{-1} & \\
& =\left(\begin{array}{cc}
0 & H_{0}^{-1} \\
0 & 0
\end{array}\right)+\left(\begin{array}{c}
-H_{0}^{-1 / 2}\left(S^{*}-\lambda H_{0}^{-1 / 2}\right) \\
I
\end{array}\right) L_{1}(\lambda)^{-1}\left(I-\left(S-\lambda H_{0}^{-1 / 2}\right) H_{0}^{-1 / 2}\right) .
\end{aligned}
$$


For the proof of Theorem 4.4, we use the following simple lemma.

Lemma 4.5. If $A$ is a symmetric operator in a Krein space $\mathcal{K}$ such that there exists a point $\lambda \in \mathbb{C}$ with $\lambda, \bar{\lambda} \in \rho(A)$, then $A$ is self-adjoint in $\mathcal{K}$.

Proof. Let $\lambda \in \mathbb{C}$ with $\lambda, \bar{\lambda} \in \rho(A)$. Then $\lambda \in \rho(A) \cap \rho\left(A^{+}\right)$and the symmetry of $A$ implies that

$$
\left(A^{+}-\bar{\lambda}\right)^{-1} \supset(A-\bar{\lambda})^{-1} .
$$

Since $\bar{\lambda} \in \rho(A)$, the right-hand side is defined on all of $\mathcal{K}$. Hence, the last inclusion is in fact an equality and so $A=A^{+}$.

Proof of Theorem 4.4. First we prove that $\rho\left(L_{1}\right) \subset \rho\left(A_{2}\right)$. Let $\lambda_{0} \in \rho\left(L_{1}\right)$ and let $\boldsymbol{u}=(u v)^{\mathrm{T}} \in \mathcal{H}_{1 / 4} \oplus \mathcal{H}_{-1 / 4}$ be arbitrary. We have to show that there exists a unique element $\boldsymbol{x}=\left(\begin{array}{ll}x y & \mathrm{~T}\end{array}\right)^{\mathrm{T}} \in \mathcal{D}\left(A_{2}\right) \subset \mathcal{D}\left(H_{0}^{1 / 2}\right) \oplus \mathcal{H}$ such that $\left(A_{2}-\lambda_{0}\right) \boldsymbol{x}=\boldsymbol{u}$ or, equivalently,

$$
\begin{aligned}
\left(V-\lambda_{0}\right) x+y & =u, \\
H_{0} x+\left(V-\lambda_{0}\right) y & =v .
\end{aligned}
$$

Since $V \in L\left(\mathcal{H}_{1 / 2}, \mathcal{H}\right)$, we have $u-\left(V-\lambda_{0}\right) H_{0}^{-1} v \in \mathcal{H}$ and thus

$$
\begin{aligned}
& y:=L_{1}\left(\lambda_{0}\right)^{-1}\left(u-\left(V-\lambda_{0}\right) H_{0}^{-1} v\right) \in \mathcal{H}, \\
& x:=H_{0}^{-1}\left(v-\left(V-\lambda_{0}\right) y\right) \in \mathcal{D}\left(H_{0}^{1 / 2}\right) .
\end{aligned}
$$

Then, by the definition of $x,(4.9)$ holds. Relation (4.8) follows since

$$
\begin{aligned}
\left(V-\lambda_{0}\right) x+y & =\left(V-\lambda_{0}\right) H_{0}^{-1}\left(v-\left(V-\lambda_{0}\right) y\right)+y \\
& =\left(V-\lambda_{0}\right) H_{0}^{-1} v+\left(I-\left(V-\lambda_{0}\right) H_{0}^{-1}\left(V-\lambda_{0}\right)\right) y \\
& =\left(V-\lambda_{0}\right) H_{0}^{-1} v+\left(I-\left(V H_{0}^{-1 / 2}-\lambda_{0} H_{0}^{-1 / 2}\right)\left(H_{0}^{-1 / 2} V-\lambda_{0} H_{0}^{-1 / 2}\right)\right) y \\
& =\left(V-\lambda_{0}\right) H_{0}^{-1} v+L_{1}\left(\lambda_{0}\right) y \\
& =u
\end{aligned}
$$

here we have used the fact that $H_{0}^{-1 / 2} V=S^{*}$ according to Remark 4.1. This proves that $A_{2}-\lambda_{0}$ is surjective. In order to show that $A_{2}-\lambda_{0}$ is injective, let $\boldsymbol{x}=(x y)^{\mathrm{T}} \in$ $\mathcal{D}\left(A_{2}\right) \subset \mathcal{D}\left(H_{0}^{1 / 2}\right) \oplus \mathcal{H}$ be such that $\left(A_{2}-\lambda_{0}\right) \boldsymbol{x}=\mathbf{0}$ or, equivalently,

$$
\begin{aligned}
\left(V-\lambda_{0}\right) x+y & =0, \\
H_{0} x+\left(V-\lambda_{0}\right) y & =0 .
\end{aligned}
$$

The second relation yields $x=-H_{0}^{-1}\left(V-\lambda_{0}\right) y$. Inserting this into the first relation, we obtain $L_{1}\left(\lambda_{0}\right) y=0$. Now $y \in \mathcal{H}$ implies that $y=0$ and hence also that $x=0$.

Since $L_{1}$ is a self-adjoint operator function in $\mathcal{H}$, its resolvent set $\rho\left(L_{1}\right)$ is symmetric to $\mathbb{R}$. Hence, $\rho\left(L_{1}\right) \neq \emptyset$ implies that $A_{2}$ is self-adjoint in $\mathcal{K}_{2}$ by Lemma 4.5. 
It remains to prove the representation for $\left(A_{2}-\lambda\right)^{-1}$. From $(4.10),(4.11)$ it follows that, for $\lambda \in \rho\left(L_{1}\right)$,

$$
\left(A_{2}-\lambda\right)^{-1}=\left(\begin{array}{cc}
-H_{0}^{-1}(V-\lambda) L_{1}(\lambda)^{-1} & H_{0}^{-1}+H_{0}^{-1}(V-\lambda) L_{1}(\lambda)^{-1}(V-\lambda) H_{0}^{-1} \\
L_{1}(\lambda)^{-1} & -L_{1}(\lambda)^{-1}(V-\lambda) H_{0}^{-1}
\end{array}\right) .
$$

Using the identities

$$
(V-\lambda) H_{0}^{-1}=\left(S-\lambda H_{0}^{-1 / 2}\right) H_{0}^{-1 / 2}, \quad H_{0}^{-1}(V-\lambda)=H_{0}^{-1 / 2}\left(S^{*}-\lambda H_{0}^{-1 / 2}\right),
$$

we arrive at (4.7). Finally, we observe that the operators

$$
\begin{aligned}
&\left(\begin{array}{l}
\left.I-\left(S-\lambda H_{0}^{-1 / 2}\right) H_{0}^{-1 / 2}\right) \\
L_{1}(\lambda)^{-1}
\end{array}\right.: \mathcal{H} \rightarrow \mathcal{H} \\
&\left(\begin{array}{c}
-H_{0}^{-1 / 2}\left(S^{*}-\lambda H_{0}^{-1 / 2}\right) \\
I
\end{array}\right): \mathcal{H} \rightarrow \mathcal{H}_{1 / 4} \oplus \mathcal{H}_{-1 / 4}
\end{aligned}
$$

are bounded and so the right-hand side of (4.7) is a bounded operator in the space $\mathcal{G}_{2}=\mathcal{H}_{1 / 4} \oplus \mathcal{H}_{-1 / 4}$.

Corollary 4.6. If $\mathcal{D}\left(H_{0}^{1 / 2}\right) \subset \mathcal{D}(V)$, we have $\rho\left(L_{1}\right) \subset \rho\left(A_{1}\right) \cap \rho\left(A_{2}\right)$ and the resolvents of $A_{1}$ and $A_{2}$ coincide on the dense subset $\mathcal{K}_{1} \cap \mathcal{K}_{2}=\mathcal{H}_{1 / 4} \oplus \mathcal{H}$ of $\mathcal{K}_{1}$ and $\mathcal{K}_{2}$ :

$$
\left(A_{1}-\lambda\right)^{-1} \boldsymbol{x}=\left(A_{2}-\lambda\right)^{-1} \boldsymbol{x}, \quad \boldsymbol{x} \in \mathcal{K}_{1} \cap \mathcal{K}_{2}, \quad \lambda \in \rho\left(L_{1}\right) \subset \rho\left(A_{1}\right) \cap \rho\left(A_{2}\right) .
$$

Proof. The claim follows easily from the formulae for the resolvents of $A_{1}$ and $A_{2}$ in Proposition 3.4 and Theorem 4.4.

\section{Spectral properties of the operators $A_{1}$ and $A_{2}$}

In this section we investigate the spectral properties of the self-adjoint operators $A_{1}$ and $A_{2}$ in the respective Krein spaces $\mathcal{K}_{1}$ and $\mathcal{K}_{2}$.

We recall that under Assumption 3.1, that is, $\mathcal{D}\left(H_{0}^{1 / 2}\right) \subset \mathcal{D}(V)$, the operator $A_{1}$ in $\mathcal{K}_{1}=\mathcal{H} \oplus \mathcal{H}$ is given by (see Theorem 3.2)

$$
\begin{aligned}
\mathcal{D}\left(A_{1}\right) & =\left\{\left(\begin{array}{l}
x \\
y
\end{array}\right) \in \mathcal{H} \oplus \mathcal{H}: x \in \mathcal{D}\left(H_{0}^{1 / 2}\right), H_{0}^{1 / 2} x+S^{*} y \in \mathcal{D}\left(H_{0}^{1 / 2}\right)\right\} \\
A_{1}\left(\begin{array}{l}
x \\
y
\end{array}\right) & =\left(\begin{array}{c}
V x+y \\
H_{0}^{1 / 2}\left(H_{0}^{1 / 2} x+S^{*} y\right)
\end{array}\right) .
\end{aligned}
$$


Under the assumption that $\rho\left(L_{1}\right) \neq \emptyset$, the operator $A_{2}$ in $\mathcal{K}_{2}=\mathcal{H}_{1 / 4} \oplus \mathcal{H}_{-1 / 4}$ is given by (see (4.6) and Remark 4.2)

$$
\begin{aligned}
\mathcal{D}\left(A_{2}\right) & =\left\{\left(\begin{array}{l}
x \\
y
\end{array}\right) \in \mathcal{H}_{1 / 4} \oplus \mathcal{H}_{-1 / 4}: x \in \mathcal{D}\left(H_{0}^{1 / 2}\right), y \in \mathcal{H},\right. \\
\left.V x+y \in \mathcal{H}_{1 / 4}, H_{0} x+V y \in \mathcal{H}_{-1 / 4}\right\} & \\
& =\left\{\left(\begin{array}{c}
x \\
y
\end{array}\right) \in \mathcal{H}_{1 / 4} \oplus \mathcal{H}_{-1 / 4}: y \in \mathcal{H}, V x+y \in \mathcal{H}_{1 / 4}, H_{0} x+V y \in \mathcal{H}_{-1 / 4}\right\}, \\
A_{2}\left(\begin{array}{l}
x \\
y
\end{array}\right) & =\left(\begin{array}{c}
V x+y \\
H_{0} x+V y
\end{array}\right) .
\end{aligned}
$$

The definitions of $A_{1}$ and $A_{2}$ imply that, for $\boldsymbol{x}=(x y)^{\mathrm{T}} \in \mathcal{D}\left(A_{j}\right) \subset \mathcal{D}\left(H_{0}^{1 / 2}\right) \oplus \mathcal{H}$,

$$
\left[A_{j} \boldsymbol{x}, \boldsymbol{x}\right]=\|y+S \hat{x}\|^{2}+\left(\left(I-S^{*} S\right) \hat{x}, \hat{x}\right), \quad j=1,2,
$$

with $\hat{x}:=H_{0}^{1 / 2} x$ in $\mathcal{H}$. Indeed, using $S \hat{x}=V x$, we obtain

$$
\begin{aligned}
{\left[A_{2} \boldsymbol{x}, \boldsymbol{x}\right] } & =(V x+y, y)+\left(H_{0} x+V y, x\right) \\
& =\left\|H_{0}^{1 / 2} x\right\|^{2}+\|y\|^{2}+(V x, y)+(y, V x) \\
& =\left\|H_{0}^{1 / 2} x\right\|^{2}+\|y\|^{2}+(S \hat{x}, y)+(y, S \hat{x}) \\
& =\|y+S \hat{x}\|^{2}+\left(\left(I-S^{*} S\right) \hat{x}, \hat{x}\right) ;
\end{aligned}
$$

the proof for $A_{1}$ is similar.

Relation (5.1) shows that the number of negative squares of the Hermitian forms $\left[A_{j} \boldsymbol{x}, \boldsymbol{y}\right], \boldsymbol{x}, \boldsymbol{y} \in \mathcal{D}\left(A_{j}\right), j=1,2$, coincides with the dimension of the spectral subspace of the self-adjoint operator $I-S^{*} S$ in $\mathcal{H}$ corresponding to the negative half-axis. The following assumption is crucial for the remaining part of this paper; it guarantees that this number is finite.

Assumption 5.1. $S=V H_{0}^{-1 / 2}=S_{0}+S_{1}$ with $\left\|S_{0}\right\|<1$ and $S_{1}$ compact in $\mathcal{H}$.

Obviously, such a decomposition of $S$ is not unique, and the operator $S_{1}$ can be chosen to have some more particular properties.

Lemma 5.2. In Assumption 5.1, without loss of generality, we can suppose that $S_{1}=\sum_{i=1}^{n}\left(\cdot, w_{i}\right) v_{i}$ with $v_{i} \in \mathcal{H}$ and $w_{i} \in \mathcal{D}\left(H_{0}^{1 / 2}\right), i=1, \ldots, n$.

Proof. Since a compact operator is the sum of an operator with arbitrarily small norm and an operator of finite rank, $S_{1}$ can be chosen to be of finite rank, say $S_{1}=\sum_{i=1}^{n}\left(\cdot, w_{i}\right) v_{i}$ with $v_{i}, w_{i} \in \mathcal{H}, i=1, \ldots, n$. Moreover, by means of an additive perturbation of arbitrarily small norm, the elements $w_{i}$ can be chosen in the dense subset $\mathcal{D}\left(H_{0}^{1 / 2}\right)$ of $\mathcal{H}$.

Lemma 5.3. If $\mathcal{D}\left(H_{0}^{1 / 2}\right) \subset \mathcal{D}(V)$ and $S=V H_{0}^{-1 / 2}$ can be decomposed as $S=S_{0}+S_{1}$ with $\left\|S_{0}\right\|<1$ and $S_{1}$ compact, then $\rho\left(L_{1}\right) \neq \emptyset$. 
Proof. According to the assumption on $S$, for $\mu \in \mathbb{R}$ the operator $L_{1}(\mathrm{i} \mu)$ can be written as

$$
L_{1}(\mathrm{i} \mu)=\left(I+\mu^{2} H_{0}^{-1}\right)^{1 / 2}(F(\mu)+K(\mu)+\mathrm{i} G(\mu))\left(I+\mu^{2} H_{0}^{-1}\right)^{1 / 2}
$$

with the bounded self-adjoint operators

$$
\begin{aligned}
& F(\mu):=I-\left(I+\mu^{2} H_{0}^{-1}\right)^{-1 / 2} S_{0} S_{0}^{*}\left(I+\mu^{2} H_{0}^{-1}\right)^{-1 / 2} \\
& K(\mu):=-\left(I+\mu^{2} H_{0}^{-1}\right)^{-1 / 2}\left(S_{0} S_{1}^{*}+S_{1} S_{0}^{*}+S_{1} S_{1}^{*}\right)\left(I+\mu^{2} H_{0}^{-1}\right)^{-1 / 2}, \\
& G(\mu):=\mu\left(I+\mu^{2} H_{0}^{-1}\right)^{-1 / 2}\left(S H_{0}^{-1 / 2}+H_{0}^{-1 / 2} S^{*}\right)\left(I+\mu^{2} H_{0}^{-1}\right)^{-1 / 2} .
\end{aligned}
$$

By (5.2), i $\mu \in \rho\left(L_{1}\right)$ if and only if $0 \in \rho(F(\mu)+K(\mu)+\mathrm{i} G(\mu))$. Since $\left\|S_{0}\right\|<1$ and $0 \leqslant\left(I+\mu^{2} H_{0}^{-1}\right)^{-1} \leqslant I$, we have $F(\mu) \geqslant I-S_{0} S_{0}^{*} \geqslant \gamma$ with some $\gamma>0$ for all $\mu \in \mathbb{R}$. The self-adjointness of $G(\mu)$ implies that $0 \in \rho(F(\mu)+\mathrm{i} G(\mu))$ for all $\mu \in \mathbb{R}$, and the claim now follows if we show that $\|K(\mu)\|<\gamma$ for $\mu \in \mathbb{R}$ sufficiently large.

To this end we observe that, for $x \in \mathcal{H}$,

$$
\left\|\left(I+\mu^{2} H_{0}^{-1}\right)^{-1 / 2} x\right\|^{2}=\int_{0}^{\left\|H_{0}^{-1}\right\|} \frac{1}{1+\mu^{2} t} \mathrm{~d}(E(t) x, x) \rightarrow 0, \quad \mu \rightarrow \infty,
$$

where $E$ is the spectral function of $H_{0}^{-1}$. Hence, the rightmost factor in (5.3) tends to 0 strongly for $\mu \rightarrow \infty$. The middle factor in (5.3) is compact since $S_{1}$ is compact and the leftmost factor is uniformly bounded for all $\mu$. Therefore, $\|K(\mu)\| \rightarrow 0$ for $\mu \rightarrow \infty$ (see, for example, $[\mathbf{5 1}, \S 6.1])$.

Lemma 5.4. Suppose that $\mathcal{D}\left(H_{0}^{1 / 2}\right) \subset \mathcal{D}(V)$ and $S=V H_{0}^{-1 / 2}$ can be decomposed as $S=S_{0}+S_{1}$ with $\left\|S_{0}\right\|<1$ and $S_{1}$ compact. Then the number of negative squares of the Hermitian form $\left[A_{1} \boldsymbol{x}, \boldsymbol{y}\right], \boldsymbol{x}, \boldsymbol{y} \in \mathcal{D}\left(A_{1}\right)$, in $\mathcal{H}_{1}$ and of the Hermitian form $\left[A_{2} \boldsymbol{x}, \boldsymbol{y}\right]$, $\boldsymbol{x}, \boldsymbol{y} \in \mathcal{D}\left(A_{2}\right)$, in $\mathcal{H}_{2}$ is finite; it is equal to the number $\kappa$ of negative eigenvalues of $I-S^{*} S$ counted with multiplicities.

Proof. Both claims follow from relation (5.1) and from the fact that, due to the assumption on $S$,

$$
I-S^{*} S=I-S_{0}^{*} S_{0}+K
$$

with a compact operator $K$. Observe that Lemma 5.3 implies that $\rho\left(L_{1}\right) \neq \emptyset$ so that $A_{2}$ is self-adjoint by Theorem 4.4.

In the following, we first consider the particular case $\|S\|<1$, which means that the operator $I-S^{*} S$ is uniformly positive. Recall that $m>0$ is such that $H_{0} \geqslant m^{2}$.

Lemma 5.5. If $\mathcal{D}\left(H_{0}^{1 / 2}\right) \subset \mathcal{D}(V)$ and $\|S\|<1$, then

$$
\sigma\left(L_{1}\right) \subset \mathbb{R} \backslash(-\alpha, \alpha),
$$

where $\alpha:=(1-\|S\|) m$. 
Proof. In the proof we use the numerical range $W\left(L_{1}\right)$ of the operator polynomial $L_{1}$ in (3.8). By definition, $W\left(L_{1}\right)$ consists of all points $\lambda \in \mathbb{C}$ for which there exists an element $x \in \mathcal{H}, x \neq 0$, such that $\left(L_{1}(\lambda) x, x\right)=0$. Since $0 \in \rho\left(L_{1}\right)$, we have $\sigma\left(L_{1}\right) \subset \overline{W\left(L_{1}\right)}$ (see [30, Theorem 26.6]). Hence, it is sufficient to show that $W\left(L_{1}\right)$ is real and does not contain points of the interval $(-\alpha, \alpha)$. The first claim follows from the fact that, for arbitrary $x \in \mathcal{H}$ with $\|x\|=1$, the quadratic polynomial

$$
\left(L_{1}(\lambda) x, x\right)=\|x\|^{2}-\left(\left(S^{*}-\lambda H_{0}^{-1 / 2}\right) x,\left(S^{*}-\bar{\lambda} H_{0}^{-1 / 2}\right) x\right)
$$

is positive at $\lambda=0$ since $\|S\|<1$ and tends to $-\infty$ if $\lambda \rightarrow \pm \infty$. For the second claim, using $\left\|H_{0}^{-1 / 2}\right\| \leqslant 1 / m$, we obtain that, for $|\lambda|<\alpha$,

$$
\begin{aligned}
\left|\left(L_{1}(\lambda) x, x\right)\right| & \geqslant\|x\|^{2}-\left\|\left(S^{*}-\lambda H_{0}^{-1 / 2}\right) x\right\|^{2} \\
& \geqslant 1-\left(\|S\|+\frac{|\lambda|}{m}\right)^{2} \\
& >1-\left(\|S\|+\frac{\alpha}{m}\right)^{2} \\
& \geqslant 0,
\end{aligned}
$$

and hence $\lambda \notin W\left(L_{1}\right)$.

The above lemma can be used to obtain information about the essential spectrum of $L_{1}$ in the more general situation of Assumption 5.1.

Lemma 5.6. If $\mathcal{D}\left(H_{0}^{1 / 2}\right) \subset \mathcal{D}(V)$ and $S=V H_{0}^{-1 / 2}$ can be decomposed as $S=S_{0}+S_{1}$ with $\left\|S_{0}\right\|<1$ and $S_{1}$ compact, then

$$
\sigma_{\text {ess }}\left(L_{1}\right) \subset \mathbb{R} \backslash(-\alpha, \alpha)
$$

where $\alpha:=\left(1-\left\|S_{0}\right\|\right) m$.

Proof. By the assumption on $S$, the operator function $L_{1}$ can be written as

$$
L_{1}(\lambda)=L_{0}(\lambda)-K(\lambda), \quad \lambda \in \mathbb{C} ;
$$

here the operator function $L_{0}$ is given by

$$
L_{0}(\lambda):=I-\left(S_{0}-\lambda H_{0}^{-1 / 2}\right)\left(S_{0}^{*}-\lambda H_{0}^{-1 / 2}\right), \quad \lambda \in \mathbb{C},
$$

and

$$
K(\lambda):=S_{1}\left(S_{0}^{*}-\lambda H_{0}^{-1 / 2}\right)+\left(S_{0}-\lambda H_{0}^{-1 / 2}\right) S_{1}^{*}
$$

is compact for all $\lambda \in \mathbb{C}$ since $S_{1}$ is compact. By Lemma 5.5 applied to $L_{0}$, we have $\sigma\left(L_{0}\right) \subset \mathbb{R} \backslash(-\alpha, \alpha)$. Hence, $\sigma\left(L_{0}\right)$ has empty interior as a subset of $\mathbb{C}$ and $\mathbb{C} \backslash \sigma\left(L_{0}\right)$ consists of only one component. By the proof of Lemma 5.3, this component contains points i $\mu \in \rho\left(L_{1}\right)$ for $\mu \in \mathbb{R}$ sufficiently large. Now [40, Lemma XIII.4] shows that

$$
\sigma_{\mathrm{ess}}\left(L_{1}\right)=\sigma_{\mathrm{ess}}\left(L_{0}\right) \subset \mathbb{R} \backslash(-\alpha, \alpha) .
$$


Theorem 5.7. Suppose that $\mathcal{D}\left(H_{0}^{1 / 2}\right) \subset \mathcal{D}(V)$ and that the operator $S=V H_{0}^{-1 / 2}$ can be decomposed as $S=S_{0}+S_{1}$ with $\left\|S_{0}\right\|<1$ and $S_{1}$ compact. Let $m>0$ be such that $H_{0} \geqslant m^{2}$ and let $\kappa$ be the number of negative eigenvalues of $I-S^{*} S$ counted with multiplicities. Then the following statements hold.

(i) The self-adjoint operator $A_{1}$ is definitizable in the Krein space $\mathcal{K}_{1}$.

(ii) The non-real spectrum of $A_{1}$ is symmetric to the real axis and consists of at most $\kappa$ pairs of eigenvalues $\lambda, \bar{\lambda}$ of finite type; the algebraic eigenspaces corresponding to $\lambda$ and $\bar{\lambda}$ are isomorphic.

(iii) For the essential spectrum of $A_{1}$ we have, with $\alpha:=\left(1-\left\|S_{0}\right\|\right) m$,

$$
\sigma_{\mathrm{ess}}\left(A_{1}\right) \subset \mathbb{R} \backslash(-\alpha, \alpha) .
$$

(iv) If $V$ is $H_{0}^{1 / 2}$-compact, then

$$
\sigma_{\text {ess }}\left(A_{1}\right)=\left\{\lambda \in \mathbb{R}: \lambda^{2} \in \sigma_{\text {ess }}\left(H_{0}\right)\right\} \subset \mathbb{R} \backslash(-m, m) .
$$

(v) If $\left\|V H_{0}^{-1 / 2}\right\|<1$, then the operator $A_{1}$ is uniformly positive in the Krein space $\mathcal{K}_{1}$ and, with $\alpha:=\left(1-\left\|V H_{0}^{-1 / 2}\right\|\right) m$,

$$
\sigma\left(A_{1}\right) \subset \mathbb{R} \backslash(-\alpha, \alpha) .
$$

Corollary 5.8. If, in addition to the assumptions of Theorem 5.7, $1 \notin \sigma_{\mathrm{p}}\left(S^{*} S\right)$ or, equivalently, $0 \notin \sigma_{\mathrm{p}}\left(A_{1}\right)$, then

$$
\kappa=\sum_{\lambda \in \sigma_{\mathrm{p}}\left(A_{1}\right) \cap(0,+\infty)} \kappa_{\lambda}^{-}\left(A_{1}\right)+\sum_{\lambda \in \sigma_{\mathrm{p}}\left(A_{1}\right) \cap(-\infty, 0)} \kappa_{\lambda}^{+}\left(A_{1}\right)+\sum_{\lambda \in \sigma_{\mathrm{p}}\left(A_{1}\right) \cap \mathbb{C}^{+}} \kappa_{\lambda}^{\mathrm{o}}\left(A_{1}\right)
$$

(see (2.4)). As a consequence, the following are true.

(i) The number of positive eigenvalues of $A_{1}$ that have a non-positive eigenvector plus the number of negative eigenvalues of $A_{1}$ that have a non-negative eigenvector plus the number of all eigenvalues of $A_{1}$ in the open upper half-plane $\mathbb{C}^{+}$is at most $\kappa$.

(ii) With the exception of the real eigenvalues in (i), the spectrum of $A_{1}$ on the positive half-axis is of positive type and the spectrum of $A_{1}$ on the negative half-axis is of negative type.

(iii) If $\left\|V H_{0}^{-1 / 2}\right\|<1$, that is, $\kappa=0$, then all the spectrum of $A_{1}$ on the positive halfaxis is of positive type and all the spectrum of $A_{1}$ on the negative half-axis is of negative type.

(iv) If $\kappa \geqslant 1$, there exists at least one eigenvalue with the properties mentioned in (i) and, in particular, $A_{1}$ has at least one eigenvalue.

Proof of Theorem 5.7. (i) We have $\rho\left(L_{1}\right) \neq \emptyset$ by Lemma 5.3, and $\rho\left(L_{1}\right) \subset \rho\left(A_{1}\right)$ by Proposition 3.4. Thus, $\rho\left(A_{1}\right) \neq \emptyset$ and hence the claim follows from Lemma 5.4 and $[\mathbf{2 4}$, Chapter I.3] (see $\S 2.3)$.

(ii) All claims follow from the definitizability of $A_{1}$ (see (i) and $\S 2.3$ ). 
For the proof of the remaining statements, we observe that, by (ii), $\sigma\left(A_{1}\right)$ has empty interior as a subset of $\mathbb{C}$. From Proposition 3.4 it follows that

$$
\left(L_{1}(\lambda)^{-1} x, y\right)=\left[\left(A_{1}-\lambda\right)^{-1}\left(\begin{array}{l}
x \\
0
\end{array}\right),\left(\begin{array}{l}
0 \\
y
\end{array}\right)\right], \quad x, y \in \mathcal{H}, \lambda \in \rho\left(L_{1}\right) .
$$

This shows that the analytic operator function $L_{1}(\cdot)^{-1}$ on $\rho\left(L_{1}\right)$ can be continued analytically to $\rho\left(A_{1}\right)$ and hence $\rho\left(A_{1}\right) \subset \rho\left(L_{1}\right)$. Since $\rho\left(L_{1}\right) \subset \rho\left(A_{1}\right)$ by Proposition 3.4, we arrive at

$$
\rho\left(A_{1}\right)=\rho\left(L_{1}\right) .
$$

The relation (5.8) also implies that if $\lambda_{0} \notin \sigma_{\text {ess }}\left(A_{1}\right)$, and hence $\left(A_{1}-\cdot\right)^{-1}$ is a finitely meromorphic operator function in a neighbourhood of $\lambda_{0}$, then so is $L_{1}(\cdot)^{-1}$, that is, $\lambda_{0} \notin \sigma_{\text {ess }}\left(L_{1}\right)$. Since $\sigma_{\text {ess }}\left(A_{1}\right) \subset \sigma_{\text {ess }}\left(L_{1}\right)$ by $(3.10)$, we obtain

$$
\sigma_{\text {ess }}\left(A_{1}\right)=\sigma_{\text {ess }}\left(L_{1}\right) \text {. }
$$

(iii) By (5.10) and Lemma 5.6 we have

$$
\sigma_{\mathrm{ess}}\left(A_{1}\right)=\sigma_{\mathrm{ess}}\left(L_{1}\right) \subset \mathbb{R} \backslash(-\alpha, \alpha) .
$$

(iv) If $V$ is $H_{0}^{1 / 2}$-compact, we can choose $S_{0}=0$, and so (5.4) and (5.5) yield that $L_{1}(\lambda)=L_{0}(\lambda)+K(\lambda)$, where $K(\lambda)$ is compact and $L_{0}$ is now given by

$$
L_{0}(\lambda):=I-\lambda^{2} H_{0}^{-1}, \quad \lambda \in \mathbb{C} .
$$

Together with (5.10) and (5.6), we obtain that

$$
\sigma_{\text {ess }}\left(A_{1}\right)=\sigma_{\text {ess }}\left(L_{1}\right)=\sigma_{\text {ess }}\left(L_{0}\right)=\left\{\lambda \in \mathbb{R}: \lambda^{2} \in \sigma_{\text {ess }}\left(H_{0}\right)\right\} .
$$

(v) If $\|S\|=\left\|V H_{0}^{-1 / 2}\right\|<1$, then equality (5.9) and Lemma 5.5 show that

$$
\sigma\left(A_{1}\right)=\sigma\left(L_{1}\right) \subset \mathbb{R} \backslash(-\alpha, \alpha) .
$$

Theorem 5.9. Suppose that $\mathcal{D}\left(H_{0}^{1 / 2}\right) \subset \mathcal{D}(V)$ and that the operator $S=V H_{0}^{-1 / 2}$ can be decomposed as $S=S_{0}+S_{1}$ with $\left\|S_{0}\right\|<1$ and $S_{1}$ compact. Let $m>0$ be such that $H_{0} \geqslant m^{2}$ and let $\kappa$ be the number of negative eigenvalues of $I-S^{*} S$ counted with multiplicities. Then the following statements hold.

(i) The self-adjoint operator $A_{2}$ is definitizable in the Krein space $\mathcal{K}_{2}$.

(ii) The spectrum, essential spectrum and point spectrum of $A_{1}$ and $A_{2}$ coincide:

$$
\sigma\left(A_{1}\right)=\sigma\left(A_{2}\right), \quad \sigma_{\mathrm{ess}}\left(A_{1}\right)=\sigma_{\mathrm{ess}}\left(A_{2}\right), \quad \sigma_{\mathrm{p}}\left(A_{1}\right)=\sigma_{\mathrm{p}}\left(A_{2}\right) ;
$$

moreover, $A_{1}$ and $A_{2}$ have the same Jordan chains and their spectral points are of the same (positive or negative) type.

(iii) The statements (ii)-(v) of Theorem 5.7 continue to hold for $A_{2}$. 
Remark 5.10. Although $A_{1}$ and $A_{2}$ have the same spectra, there is an essential difference in the behaviour of their spectral functions at $\infty$ (see $\S 6)$.

Proof of Theorem 5.9. (i) We have $\rho\left(L_{1}\right) \neq \emptyset$ by Lemma 5.3, and $\rho\left(L_{1}\right) \subset \rho\left(A_{2}\right)$ by Theorem 4.4. Thus, $\rho\left(A_{2}\right) \neq \emptyset$ and hence the claim follows from Lemma 5.4 and $[\mathbf{2 4}$, Chapter I.3] (see $\S 2.3$ ).

(ii) First we observe that, by (5.9) and Theorem 4.4, we have

$$
\rho\left(A_{1}\right)=\rho\left(L_{1}\right) \subset \rho\left(A_{2}\right)
$$

and that for $\lambda \in \rho\left(A_{1}\right)$, by (4.12),

$$
\left[\left(A_{1}-\lambda\right)^{-1} \boldsymbol{x}, \boldsymbol{y}\right]=\left[\left(A_{2}-\lambda\right)^{-1} \boldsymbol{x}, \boldsymbol{y}\right], \quad \boldsymbol{x}, \boldsymbol{y} \in \mathcal{K}_{1} \cap \mathcal{K}_{2}=\mathcal{H}_{1 / 4} \oplus \mathcal{H}
$$

If $\lambda_{0}$ is an eigenvalue of finite type of $A_{1}$, that is, $\lambda_{0} \in \sigma_{\mathrm{d}}\left(A_{1}\right)$, then it is either an isolated eigenvalue of $A_{2}$ or it belongs to $\rho\left(A_{2}\right)$ by (5.12). Then, for $j=1,2$, the corresponding Riesz projection is

$$
P_{\lambda_{0}, j}:=-\frac{1}{2 \pi \mathrm{i}} \int_{\mathcal{C}_{\lambda_{0}}}\left(A_{j}-z\right)^{-1} \mathrm{~d} z
$$

where $\mathcal{C}_{\lambda_{0}}$ is a closed Jordan curve in $\rho\left(A_{1}\right)$ surrounding $\lambda_{0}$ and no other point of the spectra of $A_{1}$ and $A_{2}$. Its range is the algebraic eigenspace of $A_{j}$ at $\lambda_{0}$ and the Jordan structure of $A_{j}$ in $\lambda_{0}$ is determined by the coefficients of the principal part of the Laurent series of $\left(A_{j}-\lambda\right)^{-1}$, given by

$$
\frac{1}{\lambda-\lambda_{0}} P_{\lambda_{0}, j}+\sum_{k=1}^{p_{j}-1} \frac{1}{\left(\lambda-\lambda_{0}\right)^{k+1}} B_{j}^{k},
$$

where $p_{j} \in \mathbb{N} \cup\{\infty\}$ and $B_{j}:=\left(A_{j}-\lambda_{0}\right) P_{\lambda_{0}, j}$ (see [15, Chapter XV.2]). Since $\lambda_{0}$ was assumed to be an eigenvalue of finite type of $A_{1}$, the projection $P_{\lambda_{0}, 1}$ is finite dimensional, $p_{1}$ is finite and $B_{1}$ is a finite rank operator. By (5.13), we have

$$
\left[A_{1}^{k} P_{\lambda_{0}, 1} \boldsymbol{x}, \boldsymbol{y}\right]=\left[A_{2}^{k} P_{\lambda_{0}, 2} \boldsymbol{x}, \boldsymbol{y}\right], \quad \boldsymbol{x}, \boldsymbol{y} \in \mathcal{K}_{1} \cap \mathcal{K}_{2} .
$$

Since $\mathcal{K}_{1} \cap \mathcal{K}_{2}=\mathcal{H}_{1 / 4} \oplus \mathcal{H}$ is dense in $\mathcal{K}_{1}=\mathcal{H} \oplus \mathcal{H}$ and in $\mathcal{K}_{2}=\mathcal{H}_{1 / 4} \oplus \mathcal{H}_{-1 / 4}$, the coefficients of the principal parts in the Laurent expansions of $\left(A_{1}-\lambda\right)^{-1}$ and $\left(A_{2}-\lambda\right)^{-1}$ at $\lambda_{0}$ coincide. Hence, we have shown that

$$
\sigma_{\mathrm{d}}\left(A_{1}\right) \subset \sigma_{\mathrm{d}}\left(A_{2}\right) .
$$

Since $A_{1}$ and $A_{2}$ are definitizable, we have

$$
\rho\left(A_{j}\right) \cup \sigma_{\mathrm{d}}\left(A_{j}\right) \cup \sigma_{\mathrm{ess}}\left(A_{j}\right)=\mathbb{C}, \quad j=1,2 .
$$

This, together with (5.12) and (5.15), implies that $\sigma_{\text {ess }}\left(A_{2}\right) \subset \sigma_{\text {ess }}\left(A_{1}\right) \subset \mathbb{R}$. It remains to be shown that

$$
\sigma_{\text {ess }}\left(A_{1}\right) \subset \sigma_{\text {ess }}\left(A_{2}\right) .
$$


Since the essential spectra of $A_{1}$ and $A_{2}$ are both real, the spectral projections $E_{j}$ of $A_{j}$ can be represented by means of contour integrals over the resolvent as follows (see $[\mathbf{2 4}$, Chapter I.3]): for $j=1,2$ and a bounded interval $\Gamma \subset \mathbb{R}$ which is admissible for $A_{j}$ and has end points $a, b, a \leqslant b$, consider the operator

$$
\hat{E}_{j}(\Gamma):=-\frac{1}{2 \pi \mathrm{i}} \int_{\mathcal{C}_{\Gamma}}^{\prime}\left(A_{j}-z\right)^{-1} \mathrm{~d} z .
$$

Here $\mathcal{C}_{\Gamma}$ is the positively oriented rectangle with corners $a+\mathrm{i} \varepsilon, b+\mathrm{i} \varepsilon, b-\mathrm{i} \varepsilon$ and $a-\mathrm{i} \varepsilon$ for $\varepsilon>0$ so small that $\mathcal{C}_{\Gamma}$ does not surround any non-real spectral points of $A_{1}$ and $A_{2}$, and the prime denotes the Cauchy principal value of the integral at $a$ and $b$. If the end points $a, b$ of $\Gamma$ are not eigenvalues of $A_{j}$, then $\hat{E}_{j}(\Gamma)=E_{j}(\Gamma)$; if $a$ is an eigenvalue of $A_{j}$ and $b$ is not an eigenvalue of $A_{j}$, then $\hat{E}_{j}(\Gamma)=E_{j}(\Gamma)+\frac{1}{2} E_{j}(\{a\})$, and similarly in all other cases for $a$ and $b$. In any case, the operators $\hat{E}_{j}(\Gamma)$ determine the spectral projections of $A_{j}$, whence

$$
\left[E_{1}(\Gamma) \boldsymbol{x}, \boldsymbol{y}\right]=\left[E_{2}(\Gamma) \boldsymbol{x}, \boldsymbol{y}\right], \quad \boldsymbol{x}, \boldsymbol{y} \in \mathcal{K}_{1} \cap \mathcal{K}_{2} .
$$

In particular, $\operatorname{dim} E_{1}(\Gamma)\left(\mathcal{K}_{1} \cap \mathcal{K}_{2}\right)=\operatorname{dim} E_{2}(\Gamma)\left(\mathcal{K}_{1} \cap \mathcal{K}_{2}\right)$ and, consequently, $E_{1}(\Gamma)$ and $E_{2}(\Gamma)$ have the same rank, which proves (5.16).

It remains to be shown that the Jordan chains of $A_{1}$ and $A_{2}$ coincide. If $\lambda_{0} \in \sigma_{\mathrm{p}}\left(A_{1}\right)$ with Jordan chain $\left(\boldsymbol{x}_{k}\right)_{k=0}^{r} \subset \mathcal{D}\left(A_{1}\right) \subset \mathcal{D}\left(H_{0}^{1 / 2}\right) \oplus \mathcal{H}, r \in \mathbb{N} \cup\{\infty\}$, then, with $\boldsymbol{x}_{-1}:=\mathbf{0}$,

$$
\left(A_{1}-\lambda_{0}\right) \boldsymbol{x}_{k}=\boldsymbol{x}_{k-1}, \quad k=0,1, \ldots, r .
$$

Hence, for $\boldsymbol{x}_{k}=:\left(x_{k} y_{k}\right)^{\mathrm{T}}$, we have $y_{k} \in \mathcal{H}$ and

$$
\left.\begin{array}{c}
V x_{k}+y_{k}=x_{k-1}+\lambda_{0} x_{k} \in \mathcal{D}\left(H_{0}^{1 / 2}\right) \subset \mathcal{H}_{1 / 4}, \\
H_{0}^{1 / 2} x_{k}+S^{*} y_{k}=H_{0}^{-1 / 2}\left(y_{k-1}+\lambda_{0} y_{k}\right) \in \mathcal{D}\left(H_{0}^{1 / 2}\right) \subset \mathcal{H}_{1 / 4},
\end{array}\right\}
$$

and thus $H_{0} x_{k}+V y_{k} \in \mathcal{H}_{-1 / 4}$. This shows that $\left(\boldsymbol{x}_{k}\right)_{k=0}^{r} \subset \mathcal{D}\left(A_{2}\right)$ and so $\left(\boldsymbol{x}_{k}\right)_{k=0}^{r}$ is a Jordan chain of $A_{2}$ at $\lambda_{0}$. Conversely, if $\left(\boldsymbol{x}_{k}\right)_{k=0}^{r} \subset \mathcal{D}\left(A_{2}\right) \subset \mathcal{D}\left(H_{0}^{1 / 2}\right) \oplus \mathcal{H}$ is a Jordan chain of $A_{2}$ at $\lambda_{0}$, then, in (5.17), the element $V x_{k}+y_{k}$ belongs to $\mathcal{D}\left(H_{0}^{1 / 2}\right) \subset \mathcal{H}$ and the element $H_{0}^{1 / 2} x_{k}+S^{*} y_{k}$ belongs to $\mathcal{D}\left(H_{0}^{1 / 2}\right)$. Thus, $\left(\boldsymbol{x}_{k}\right)_{k=0}^{r} \subset \mathcal{D}\left(A_{1}\right)$ and so $\left(\boldsymbol{x}_{k}\right)_{k=0}^{r}$ is a Jordan chain of $A_{1}$ at $\lambda_{0}$.

To conclude this section, we compare the spectral properties of $A_{1}$ with those of the operator $A$ associated with the abstract Klein-Gordon equation in [27]. This operator acts in the space $\mathcal{G}:=\mathcal{H}_{1 / 2} \oplus \mathcal{H}$; if Assumption 3.1 holds and if $1 \in \rho\left(S^{*} S\right)$, it is defined as

$$
A=\left(\begin{array}{cc}
0 & I \\
H & 2 V
\end{array}\right), \quad \mathcal{D}(A)=\mathcal{D}(H) \oplus \mathcal{D}\left(H_{0}^{1 / 2}\right),
$$

with $H:=H_{0}^{1 / 2}\left(I-S^{*} S\right) H_{0}^{1 / 2}$. The operator $A$ is related to the operator $A_{1}$ introduced in $\S 3$ by the formula

$$
A=W A_{1} W^{-1},
$$


where $W$ acts from $\mathcal{G}_{1}=\mathcal{H} \oplus \mathcal{H}$ to $\mathcal{G}=\mathcal{H}_{1 / 2} \oplus \mathcal{H}$

$$
W:=\left(\begin{array}{cc}
I & 0 \\
V & I
\end{array}\right), \quad \mathcal{D}(W):=\mathcal{H}_{1 / 2} \oplus \mathcal{H} .
$$

It was shown in $[\mathbf{2 7}]$ that the operator $A$ is self-adjoint in $\mathcal{K}:=(\mathcal{G},\langle\cdot, \cdot\rangle)$ with inner product

$$
\left\langle\boldsymbol{x}, \boldsymbol{x}^{\prime}\right\rangle=\left(\left(I-S^{*} S\right) H_{0}^{1 / 2} x, H_{0}^{1 / 2} x^{\prime}\right)+\left(y, y^{\prime}\right), \quad \boldsymbol{x}=(x y)^{\mathrm{T}}, \boldsymbol{x}^{\prime}=\left(x^{\prime} y^{\prime}\right)^{\mathrm{T}} \in \mathcal{G},
$$

which is a Pontryagin space due to Assumption 5.1. Note that the negative index of this Pontryagin space equals the number $\kappa$ of negative eigenvalues of $I-S^{*} S$ counted with multiplicities (cf. Lemma 5.4). Between the indefinite inner products $\langle\cdot, \cdot\rangle$ of $\mathcal{K}$ and $[\cdot, \cdot]$ of $\mathcal{K}_{1}$ we have the relation (see [27, Proposition 4.4(i)])

$$
\left\langle\boldsymbol{x}, \boldsymbol{x}^{\prime}\right\rangle=\left[A_{1} W^{-1} \boldsymbol{x}, W^{-1} \boldsymbol{x}^{\prime}\right], \quad \boldsymbol{x} \in W \mathcal{D}\left(A_{1}\right), \boldsymbol{x}^{\prime} \in \mathcal{G} .
$$

Theorem 5.11. Suppose that $\mathcal{D}\left(H_{0}^{1 / 2}\right) \subset \mathcal{D}(V)$, that the operator $S=V H_{0}^{-1 / 2}$ can be decomposed as $S=S_{0}+S_{1}$ with $\left\|S_{0}\right\|<1$ and $S_{1}$ compact, and that $1 \in \rho\left(S^{*} S\right)$. Then

$$
\sigma(A)=\sigma\left(A_{1}\right)=\sigma\left(A_{2}\right), \quad \sigma_{\mathrm{ess}}(A)=\sigma_{\mathrm{ess}}\left(A_{1}\right)=\sigma_{\mathrm{ess}}\left(A_{2}\right), \quad \sigma_{\mathrm{p}}(A)=\sigma_{\mathrm{p}}\left(A_{1}\right)=\sigma_{\mathrm{p}}\left(A_{2}\right) .
$$

Proof. By [27, Theorem 5.2(iii)] and Theorem 5.7 (iii), the non-real spectra of $A$ and $A_{1}$ consist of finitely many eigenvalues of finite type. If $\lambda \in \rho(A) \cap \rho\left(A_{1}\right)$, then for $\boldsymbol{w}, \boldsymbol{x}^{\prime} \in \mathcal{G}$ we obtain, from (5.20) with $\boldsymbol{x}=(A-\lambda)^{-1} \boldsymbol{w} \in \mathcal{D}(A) \subset W \mathcal{D}\left(A_{1}\right)$ and (5.19),

$$
\begin{aligned}
\left\langle(A-\lambda)^{-1} \boldsymbol{w}, \boldsymbol{x}^{\prime}\right\rangle & =\left[A_{1} W^{-1}(A-\lambda)^{-1} \boldsymbol{w}, W^{-1} \boldsymbol{x}^{\prime}\right] \\
& =\left[A_{1}\left(A_{1}-\lambda\right)^{-1} W^{-1} \boldsymbol{w}, W^{-1} \boldsymbol{x}^{\prime}\right] \\
& =\left[W^{-1} \boldsymbol{w}, W^{-1} \boldsymbol{x}^{\prime}\right]+\lambda\left[\left(A_{1}-\lambda\right)^{-1} W^{-1} \boldsymbol{w}, W^{-1} \boldsymbol{x}^{\prime}\right] .
\end{aligned}
$$

In a similar way as in the proof of Theorem 5.9, observing that the range of $W^{-1}$, which is given by $\mathcal{D}(W)=\mathcal{H}_{1 / 2} \oplus \mathcal{H}$, is dense in $\mathcal{G}_{1}=\mathcal{H} \oplus \mathcal{H}$, one can show that all eigenvalues of finite type of $A_{1}$ and $A$ and also their essential spectra coincide.

The equality of the point spectra of $A$ and $A_{1}$ follows from (5.19): if $\lambda$ is an eigenvalue of $A$ with eigenvector $x \in \mathcal{D}(A)$, then $W^{-1} x \in \mathcal{D}\left(A_{1}\right)$ and $W^{-1} x$ is an eigenvector of $A_{1}$ corresponding to the eigenvalue $\lambda$. Conversely, if $\lambda$ is an eigenvalue of $A_{1}$ with eigenvector $x \in \mathcal{D}\left(A_{1}\right)$, then $W x \in \mathcal{D}(A)$ and $W x$ is an eigenvector of $A$ corresponding to the eigenvalue $\lambda$.

The equalities with the various parts of $\sigma\left(A_{2}\right)$ follow from Theorem 5.9.

\section{The critical point $\infty: A_{2}$ as a generator of a strongly continuous unitary group}

Although the spectra of $A_{1}$ and $A_{2}$ coincide, their spectral functions $E_{1}$ and $E_{2}$ behave differently at $\infty$ if $H_{0}$ is unbounded. This will be proved first for the case $V=0$; for 
$V \neq 0$ satisfying Assumption 5.1, a perturbation theorem due to Ćurgus (see [8]) applies and shows that $\infty$ is a regular critical point for $A_{2}$.

The unperturbed operators $A_{1,0}$ in $\mathcal{G}_{1}=\mathcal{H} \oplus \mathcal{H}$ and $A_{2,0}$ in $\mathcal{G}_{2}=\mathcal{H}_{1 / 4} \oplus \mathcal{H}_{-1 / 4}$ are given by

$$
\begin{array}{ll}
A_{1,0}:=\left(\begin{array}{cc}
0 & I \\
H_{0} & 0
\end{array}\right), & \mathcal{D}\left(A_{1,0}\right):=\mathcal{D}\left(H_{0}\right) \oplus \mathcal{H}, \\
A_{2,0}:=\left(\begin{array}{cc}
0 & I \\
H_{0} & 0
\end{array}\right), & \mathcal{D}\left(A_{2,0}\right):=\mathcal{D}\left(H_{0}^{3 / 4}\right) \oplus \mathcal{D}\left(H_{0}^{1 / 4}\right) .
\end{array}
$$

In fact, if $V=0$, then the operators $A_{1}$ in $\mathcal{G}_{1}$ and $A_{2}$ in $\mathcal{G}_{2}$ coincide with the operators $A_{1,0}$ and $A_{2,0}$.

Lemma 6.1. If $H_{0}$ is unbounded, then $\infty$ is a regular critical point of $A_{2,0}$, whereas it is a singular critical point of $A_{1,0}$.

Proof. The resolvents of $A_{1,0}$ and $A_{2,0}$ are defined for $\lambda \in \mathbb{C}$ such that $\lambda^{2} \notin \sigma\left(H_{0}\right)$; they are of the form

$$
\left(A_{j, 0}-\lambda\right)^{-1}=\left(\begin{array}{cc}
\lambda\left(H_{0}-\lambda^{2}\right)^{-1} & \left(H_{0}-\lambda^{2}\right)^{-1} \\
I+\lambda^{2}\left(H_{0}-\lambda^{2}\right)^{-1} & \lambda\left(H_{0}-\lambda^{2}\right)^{-1}
\end{array}\right), \quad j=1,2 .
$$

Denote the spectral function of $H_{0}^{1 / 2}$ in $\mathcal{H}$ by $E_{0}$ and let $\Gamma \subset \mathbb{R}$ be a bounded interval with $\Gamma>0$ or $\Gamma<0$. Using the equality

$$
\left(H_{0}-\lambda^{2}\right)^{-1}=\left(H_{0}^{1 / 2}-\lambda\right)^{-1}\left(H_{0}^{1 / 2}+\lambda\right)^{-1}=\frac{1}{2 \lambda}\left(\left(H_{0}^{1 / 2}-\lambda\right)^{-1}-\left(H_{0}^{1 / 2}+\lambda\right)^{-1}\right)
$$

and observing that $\left(H_{0}^{1 / 2}-\cdot\right)^{-1}$ is holomorphic on $\Gamma$ if $\Gamma<0$ and $\left(H_{0}^{1 / 2}+\cdot\right)^{-1}$ is holomorphic on $\Gamma$ if $\Gamma>0$, we find that the spectral projection $E_{j}(\Gamma)$ of $A_{j, 0}$ in $\mathcal{K}_{j}$ for $j=1,2$ is given by

$$
E_{j}(\Gamma)= \pm \frac{1}{2}\left(\begin{array}{cc}
E_{0}(\Gamma) & H_{0}^{-1 / 2} E_{0}(\Gamma) \\
H_{0}^{1 / 2} E_{0}(\Gamma) & E_{0}(\Gamma)
\end{array}\right) .
$$

Hence, for elements $\boldsymbol{x}=(x 0)^{\mathrm{T}} \in \mathcal{G}_{1}=\mathcal{H} \oplus \mathcal{H}$, we have

$$
\left\|E_{1}(\Gamma) \boldsymbol{x}\right\|_{\mathcal{G}_{1}}^{2}=\frac{1}{4}\left(\left\|E_{0}(\Gamma) x\right\|^{2}+\left\|H_{0}^{1 / 2} E_{0}(\Gamma) x\right\|^{2}\right) .
$$

If $H_{0}$ is unbounded, the last term does not remain bounded if $\Gamma>0$ extends to $\infty$ and $x \notin \mathcal{D}\left(H_{0}^{1 / 2}\right)$.

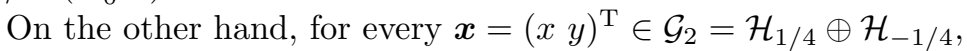

$$
\begin{aligned}
\left\|E_{2}(\Gamma) \boldsymbol{x}\right\|_{\mathcal{G}_{2}}^{2}= & \frac{1}{4}\left\|H_{0}^{1 / 4}\left(E_{0}(\Gamma) x+H_{0}^{-1 / 2} E_{0}(\Gamma) y\right)\right\|^{2} \\
& \quad+\frac{1}{4}\left\|H_{0}^{-1 / 4}\left(H_{0}^{1 / 2} E_{0}(\Gamma) x+E_{0}(\Gamma) y\right)\right\|^{2} \\
\leqslant & \left\|H_{0}^{1 / 4} x\right\|^{2}+\left\|H_{0}^{-1 / 4} y\right\|^{2} \\
= & \|\boldsymbol{x}\|_{\mathcal{G}_{2}}^{2},
\end{aligned}
$$

and therefore $\left\|E_{2}(\Gamma)\right\| \leqslant 1$. 
Since for the operator $A_{1}$ already in the unperturbed situation (that is, $V=0$ and $\left.A_{1}=A_{1,0}\right)$ the point $\infty$ is a singular critical point if $H_{0}$ is unbounded, it will remain a singular critical point for large classes of perturbations (e.g. for bounded perturbations).

For $A_{2}$, however, in the unperturbed situation (that is, $V=0$ and $A_{2}=A_{2,0}$ ) the point $\infty$ is a regular critical point. Due to Assumptions 3.1 and 5.1, we may apply a perturbation result of Ćurgus (see [8, Corollary 3.6]), which guarantees that under certain additive perturbations the critical point $\infty$ remains regular.

In order to see this, we introduce sesquilinear forms $\mathfrak{h}_{0}$ and $\mathfrak{v}$ in the Hilbert space $\mathcal{G}_{2}=\mathcal{H}_{1 / 4} \oplus \mathcal{H}_{-1 / 4}$ on $\mathcal{D}\left(\mathfrak{h}_{0}\right)=\mathcal{D}(\mathfrak{v})=\mathcal{D}\left(H_{0}^{1 / 2}\right) \oplus \mathcal{H}$ by

$$
\begin{aligned}
\mathfrak{h}_{0}(\boldsymbol{x}, \boldsymbol{y}) & :=\left(H_{0}^{1 / 2} x_{1}, H_{0}^{1 / 2} y_{1}\right)+\left(x_{2}, y_{2}\right), \\
\mathfrak{v}(\boldsymbol{x}, \boldsymbol{y}) & :=\left(V x_{1}, y_{2}\right)+\left(x_{2}, V y_{1}\right)
\end{aligned}
$$

for $\boldsymbol{x}=\left(x_{1}, x_{2}\right)^{\mathrm{T}}, \boldsymbol{y}=\left(y_{1}, y_{2}\right)^{\mathrm{T}} \in \mathcal{D}\left(H_{0}^{1 / 2}\right) \oplus \mathcal{H}$. It is not difficult to see that the form $\mathfrak{h}_{0}$ is closed, symmetric and uniformly positive. Moreover, for $\boldsymbol{x} \in \mathcal{D}\left(A_{2,0}\right), \boldsymbol{y} \in \mathcal{D}\left(\mathfrak{h}_{0}\right)$, we have

$$
\mathfrak{h}_{0}(\boldsymbol{x}, \boldsymbol{y})=\left[A_{2,0} \boldsymbol{x}, \boldsymbol{y}\right]=\left(J A_{2,0} \boldsymbol{x}, \boldsymbol{y}\right)_{\mathcal{G}_{2}},
$$

where $J$ is defined as in (4.4), [., $\cdot]$ is the indefinite inner product of $\mathcal{K}_{2}=\mathcal{H}_{1 / 4} \oplus \mathcal{H}_{-1 / 4}$ (see $(4.3))$ and $(\cdot, \cdot)_{\mathcal{G}_{2}}$ is the corresponding Hilbert space inner product.

Lemma 6.2. Let $\mathcal{D}\left(H_{0}^{1 / 2}\right) \subset \mathcal{D}(V)$ and suppose that $S=V H_{0}^{-1 / 2}$ can be decomposed as $S=S_{0}+S_{1}$ with $\left\|S_{0}\right\|<1$ and $S_{1}$ compact. Then

(i) the form $\mathfrak{v}$ is $\mathfrak{h}_{0}$-bounded with $\mathfrak{h}_{0}$-bound less than 1 ,

(ii) the form $\mathfrak{h}:=\mathfrak{h}_{0}+\mathfrak{v}$ defined on $\mathcal{D}\left(\mathfrak{h}_{0}\right)=\mathcal{D}\left(H_{0}^{1 / 2}\right) \oplus \mathcal{H}$ is closed, symmetric and bounded from below.

Proof. (i) By the assumption on $S$ and Lemma 5.2, we may choose the operator $S_{1}$ to be of the form $S_{1}=\sum_{i=1}^{n}\left(\cdot, w_{i}\right) v_{i}$ with $v_{i} \in \mathcal{H}$ and $w_{i} \in \mathcal{D}\left(H_{0}^{1 / 2}\right), i=1, \ldots, n$. Then the operator $S_{1} H_{0}^{1 / 2}$ in $\mathcal{H}$ is bounded on $\mathcal{D}\left(H_{0}^{1 / 2}\right)$ :

$$
\left\|S_{1} H_{0}^{1 / 2} x\right\| \leqslant \sum_{i=1}^{n}\left|\left(x, H_{0}^{1 / 2} w_{i}\right)\right|\left\|v_{i}\right\| \leqslant\left(\sum_{i=1}^{n}\left\|H_{0}^{1 / 2} w_{i}\right\|\left\|v_{i}\right\|\right)\|x\|=: a\|x\|
$$

for $x \in \mathcal{D}\left(H_{0}^{1 / 2}\right)$. If we set $b:=\left\|S_{0}\right\|<1$, we obtain that

$$
\|V x\|=\left\|\left(S_{0}+S_{1}\right) H_{0}^{1 / 2} x\right\| \leqslant a\|x\|+b\left\|H_{0}^{1 / 2} x\right\|, \quad x \in \mathcal{D}\left(H_{0}^{1 / 2}\right),
$$

that is, $V$ is $H_{0}^{1 / 2}$-bounded with relative bound less than 1 . It is not difficult to check (see $[\mathbf{2 1}, \S \mathrm{V} 4.1])$ that $(6.2)$ implies that there exist constants $a^{\prime}, b^{\prime} \geqslant 0, b^{\prime}<1$, such that

$$
\|V x\|^{2} \leqslant a^{\prime 2}\|x\|^{2}+{b^{\prime}}^{2}\left\|H_{0}^{1 / 2} x\right\|^{2}, \quad x \in \mathcal{D}\left(H_{0}^{1 / 2}\right) .
$$

Now let $\boldsymbol{x}=\left(x_{1}, x_{2}\right)^{\mathrm{T}} \in \mathcal{D}(\mathfrak{v})=\mathcal{D}\left(H_{0}^{1 / 2}\right) \oplus \mathcal{H}$. Using (6.3) and the inequality

$$
\left\|H_{0}^{1 / 4} x_{1}\right\|^{2}=\left|\left(H_{0}^{1 / 2} x_{1}, x_{1}\right)\right| \geqslant m\left\|x_{1}\right\|^{2}
$$


we obtain the estimate

$$
\begin{aligned}
\mathfrak{v}(\boldsymbol{x}, \boldsymbol{x}) & =2 \operatorname{Re}\left(V x_{1}, x_{2}\right) \\
& \leqslant 2\left\|V x_{1}\right\|\left\|x_{2}\right\| \\
& \leqslant \frac{1}{b^{\prime}}\left\|V x_{1}\right\|^{2}+b^{\prime}\left\|x_{2}\right\|^{2} \\
& \leqslant \frac{1}{b^{\prime}}\left(a^{\prime 2}\left\|x_{1}\right\|^{2}+{b^{\prime}}^{2}\left\|H_{0}^{1 / 2} x_{1}\right\|^{2}\right)+b^{\prime}\left\|x_{2}\right\|^{2} \\
& \leqslant \frac{{a^{\prime}}^{2}}{b^{\prime}}\left\|x_{1}\right\|^{2}+b^{\prime}\left(\left\|H_{0}^{1 / 2} x_{1}\right\|^{2}+\left\|x_{2}\right\|^{2}\right) \\
& \leqslant \frac{a^{\prime 2}}{b^{\prime} m}\left(\left\|H_{0}^{1 / 4} x_{1}\right\|^{2}+\left\|H_{0}^{-1 / 4} x_{2}\right\|^{2}\right)+b^{\prime}\left(\left\|H_{0}^{1 / 2} x_{1}\right\|^{2}+\left\|x_{2}\right\|^{2}\right) \\
& =\frac{a^{\prime 2}}{b^{\prime} m}(\boldsymbol{x}, \boldsymbol{x})_{\mathcal{G}_{2}}+b^{\prime} \mathfrak{h}_{0}(\boldsymbol{x}, \boldsymbol{x}) .
\end{aligned}
$$

(ii) It is easy to see that $\mathfrak{h}$ is symmetric since $\mathfrak{h}_{0}$ and $\mathfrak{v}$ are also symmetric. All other claims follow from the perturbation result [21, Theorem VI.1.33].

Theorem 6.3. Let $\mathcal{D}\left(H_{0}^{1 / 2}\right) \subset \mathcal{D}(V)$ and suppose that $S=V H_{0}^{-1 / 2}$ can be decomposed as $S=S_{0}+S_{1}$ with $\left\|S_{0}\right\|<1$ and $S_{1}$ compact. Then $\infty$ is a regular critical point of $A_{2}$.

Proof. According to Lemma 6.2, Assumptions (A) and (B) of $[\mathbf{9}, \S 2]$ are satisfied. By (6.1) and the first representation theorem (see [21, Theorem VI.2.1]), the operator $J A_{2,0}$ is the self-adjoint operator associated with the closed form $\mathfrak{h}_{0}$ in $\mathcal{H}_{2}$. Analogously, it follows that $J A_{2}$ is the self-adjoint operator associated with the perturbed form $\mathfrak{h}=\mathfrak{h}_{0}+\mathfrak{v}$ in $\mathcal{H}_{2}$. Since $A_{2}$ is definitizable by Theorem 5.9 and $\infty$ is a regular critical point for $A_{2,0}$ by Lemma 6.1 , it is also a regular critical point for $A_{2}$ by [9, Proposition 2.1].

Remark 6.4. Theorem 6.3 was proved in [9, Theorem 3.5] for the particular cases when either $S_{0}=0$ or $S_{1}=0$.

An important consequence of the regularity of the critical point $\infty$ is that $A_{2}$ is the generator of a unitary group in $\mathcal{K}_{2}$ and thus we obtain information on the solvability of the Cauchy problem for the differential equation

$$
\frac{\mathrm{d} \boldsymbol{x}}{\mathrm{d} t}=\mathrm{i} A_{2} \boldsymbol{x} .
$$

A function $\boldsymbol{x}: \mathbb{R} \rightarrow \mathcal{K}_{2}$ is called a classical solution of this differential equation if

$$
\boldsymbol{x} \in C^{1}\left(\mathbb{R}, \mathcal{K}_{2}\right), \quad \boldsymbol{x}(t) \in \mathcal{D}\left(A_{2}\right), \quad \frac{\mathrm{d} \boldsymbol{x}}{\mathrm{d} t}(t)=\mathrm{i} A_{2} \boldsymbol{x}(t), \quad t \in \mathbb{R} .
$$

Theorem 6.5. Let $\mathcal{D}\left(H_{0}^{1 / 2}\right) \subset \mathcal{D}(V)$ and suppose that $S=V H_{0}^{-1 / 2}$ can be decomposed as $S=S_{0}+S_{1}$ with $\left\|S_{0}\right\|<1$ and $S_{1}$ compact. Then the operator $A_{2}$ is the 
infinitesimal generator of a strongly continuous group $\left(\mathrm{e}^{\mathrm{i} t A_{2}}\right)_{t \in \mathbb{R}}$ of unitary operators in $\mathcal{K}_{2}$. If $\boldsymbol{x}_{0} \in \mathcal{D}\left(A_{2}\right)$, the Cauchy problem

$$
\frac{\mathrm{d} \boldsymbol{x}}{\mathrm{d} t}=\mathrm{i} A_{2} \boldsymbol{x}, \quad \boldsymbol{x}(0)=\boldsymbol{x}_{0},
$$

has a unique classical solution given by

$$
\boldsymbol{x}(t)=\mathrm{e}^{\mathrm{i} t A_{2}} \boldsymbol{x}_{0}, \quad t \in \mathbb{R} .
$$

Proof. The regularity of the critical point $\infty$ by Theorem 6.3 implies that $A_{2}$ is the sum of a bounded operator and an operator similar to a self-adjoint operator. As a consequence, the operators $\mathrm{e}^{\mathrm{i} t A_{2}}, t \in \mathbb{R}$, are defined and form a strongly continuous group of unitary operators in $\mathcal{K}_{2}$. The last claim follows in a similar way as corresponding results for semi-groups (see, for example, [23, Theorem 1.1]).

Remark 6.6. If only $\boldsymbol{x}_{0} \in \mathcal{K}_{2}$, then (6.5) is the unique mild solution of (6.4), that is,

$$
\boldsymbol{x} \in C\left(\mathbb{R}, \mathcal{K}_{2}\right), \quad \int_{s}^{t} \boldsymbol{x}(\tau) \mathrm{d} \tau \in \mathcal{D}\left(A_{2}\right), \quad \boldsymbol{x}(t)=\boldsymbol{x}(s)+\mathrm{i} A_{2} \int_{s}^{t} \boldsymbol{x}(\tau) \mathrm{d} \tau, \quad s, t \in \mathbb{R}
$$

(see the analogous definitions and results for semi-groups, e.g. in [1, § 1.2], [2, 3.1.12]).

\section{Semi-groups associated with $A_{1}$}

In this section we restrict ourselves to the case that the symmetric operator $V$ in $\mathcal{H}$ is everywhere defined and hence bounded. Then Assumption 3.1 used in $\S 3$ is automatically satisfied and the operator $\hat{A}_{1}$ in $\mathcal{G}_{1}=\mathcal{H} \oplus \mathcal{H}$ defined in (3.2) is already closed:

$$
A_{1}=\hat{A}_{1}=\left(\begin{array}{cc}
V & I \\
H_{0} & V
\end{array}\right), \quad \mathcal{D}\left(A_{1}\right)=\mathcal{D}\left(H_{0}\right) \oplus \mathcal{H} .
$$

Moreover, Assumption 5.1 used in $\S 5$ holds if $V$ can be decomposed as $V=V_{0}+V_{1}$ such that $\left\|V_{0}\right\|<m$ and $V_{1} H_{0}^{-1 / 2}$ is compact.

Then, according to Theorem 5.7, the operator $A_{1}$ is definitizable in $\mathcal{K}_{1}$ and the interval $\left(-\left(m-\left\|V_{0}\right\|\right), m-\left\|V_{0}\right\|\right)$ contains only eigenvalues of finite type; in particular, there exists a real point $\mu \in \rho\left(A_{1}\right)$.

Lemma 7.1. Assume that $V$ is bounded and can be decomposed as $V=V_{0}+V_{1}$ such that $\left\|V_{0}\right\|<m$ and $V_{1} H_{0}^{-1 / 2}$ is compact. Let $\kappa$ be the number of negative eigenvalues of $I-S^{*} S$ counted with multiplicities and let $\mu \in \rho\left(A_{1}\right) \cap \mathbb{R}$. There then exists a maximal non-negative subspace $\mathcal{L}_{+} \subset \mathcal{K}_{1}$ that is invariant under $\left(A_{1}-\mu\right)^{-1}$ and such that

$$
\operatorname{Im} \sigma\left(\left.\left(A_{1}-\mu\right)^{-1}\right|_{\mathcal{L}_{+}}\right) \leqslant 0 .
$$

The subspace $\mathcal{L}_{+}$can be chosen so that it contains all the algebraic eigenspaces of $A_{1}$ corresponding to the eigenvalues in the open upper half-plane, all the positive spectral subspaces and a non-negative eigenvector of $A_{1}$ at all real eigenvalues of $A_{1}$ that are not of negative type; the negative spectral subspaces of $A_{1}$ are orthogonal to $\mathcal{L}_{+}$. Furthermore,

$$
\operatorname{dim} \mathcal{L}_{+} \cap \mathcal{L}_{+}^{[\perp]} \leqslant \kappa
$$


Remark 7.2. For $z$ in a complex neighbourhood of $\mu$, the relation

$$
\left(A_{1}-z\right)^{-1}=\sum_{j=0}^{\infty}(z-\mu)^{j}\left(A_{1}-\mu\right)^{-j-1}
$$

holds and implies that the subspace $\mathcal{L}_{+}$from Lemma 7.1 is also invariant under $\left(A_{1}-z\right)^{-1}$. Since $\rho\left(A_{1}\right)$ is connected, an analytic continuation argument shows that this continues to hold for all $z \in \rho\left(A_{1}\right)$.

Proof of Lemma 7.1. Since $\left(A_{1}-\mu\right)^{-1}$ is a bounded definitizable operator, the existence of a maximal non-negative invariant subspace $\mathcal{L}_{+}^{0}$ for $\left(A_{1}-\mu\right)^{-1}$ follows from [24, Satz 3.2]. If $\lambda_{0} \in \mathbb{C}^{+}$is an eigenvalue of $A_{1}$ with algebraic eigenspace $\mathcal{L}_{\lambda_{0}}\left(A_{1}\right)$, then, together with $\mathcal{L}_{+}^{0}$, the subspace

$$
\mathcal{L}_{+}^{1}:=\mathcal{L}_{+}^{0} \cap\left(\mathcal{L}_{\lambda_{0}}\left(A_{1}\right)+\mathcal{L}_{\overline{\lambda_{0}}}\left(A_{1}\right)\right)^{[\perp]} \dot{+} \mathcal{L}_{\lambda_{0}}\left(A_{1}\right)
$$

is also a maximal non-negative invariant subspace for $\left(A_{1}-\mu\right)^{-1}$ and it contains $\mathcal{L}_{\lambda_{0}}\left(A_{1}\right)$. Since $A_{1}$ has only a finite number of non-real eigenvalues, after repeating this argument a finite number of times, the new subspace $\mathcal{L}_{+}:=\mathcal{L}_{+}^{n}$ contains all the algebraic eigenspaces of $A_{1}$ corresponding to eigenvalues in the open upper half-plane. If $\mathcal{L}_{+}$does not contain all positive subspaces of the form $E_{1}(\Gamma) \mathcal{K}_{1}$, adding such a subspace to $\mathcal{L}_{+}$would still give a non-negative invariant subspace, a contradiction to the maximality of $\mathcal{L}_{+}$. In a similar way, it can be shown that it contains non-negative eigenelements of $A_{1}$ at all real eigenvalues of $A_{1}$ that are not of negative type. Finally, the subspace on the left-hand side of $(7.1)$ is neutral with respect to the inner product $\left[A_{1} \cdot, \cdot\right]$ and hence of dimension less than or equal to $\kappa$.

Lemma 7.3. If $\mathcal{L}_{+}$is a maximal non-negative subspace as in Lemma 7.1, then

$$
\left(\begin{array}{l}
0 \\
y
\end{array}\right) \in \mathcal{L}_{+} \quad \Longrightarrow \quad y=0
$$

Proof. It is easy to see that the resolvent of $A_{1}$ admits the representation

$$
\left(A_{1}-z\right)^{-1}=\left(\begin{array}{cc}
-D(z)^{-1}(V-z) & D(z)^{-1} \\
I+(V-z) D(z)^{-1}(V-z) & -(V-z) D(z)^{-1}
\end{array}\right), \quad z \in \rho\left(A_{1}\right),
$$

where $D(z):=H_{0}-(V-z)^{2}, z \in \mathbb{C}$. For any $z \in \rho\left(A_{1}\right)$, we have $\left(A_{1}-z\right)^{-1} \mathcal{L}_{+} \subset \mathcal{L}_{+}$ which implies that $\left(A_{1}-\bar{z}\right)^{-1} \mathcal{L}_{+}^{[\perp]} \subset \mathcal{L}_{+}^{[\perp]}$. Hence,

$$
\left(A_{1}-z\right)^{-1}\left(\mathcal{L}_{+} \cap \mathcal{L}_{+}^{[\perp]}\right) \subset \mathcal{L}_{+} \cap \mathcal{L}_{+}^{[\perp]}, \quad z \in \rho\left(A_{1}\right),
$$

that is, $\left(A_{1}-z\right)^{-1}$ maps the isotropic subspace of $\mathcal{L}_{+}$into itself. Since an element $\boldsymbol{x}=(0 y)^{\mathrm{T}} \in \mathcal{L}_{+}$as in $(7.2)$ is neutral in $\mathcal{K}_{1}$, we have $\boldsymbol{x} \in \mathcal{L}_{+} \cap \mathcal{L}_{+}^{[\perp]}$ and so

$$
0=\left[\left(A_{1}-z\right)^{-1} \boldsymbol{x},\left(A_{1}-z\right)^{-1} \boldsymbol{x}\right]=-2\left((V-\operatorname{Re} z) D(z)^{-1} y, D(z)^{-1} y\right) .
$$

Choosing $z \in \mathbb{C}$ such that $V-\operatorname{Re} z>0$, we obtain $y=0$. 
Lemma 7.3 shows that $\mathcal{L}_{+}$is the graph of a closed linear operator $K$ in $\mathcal{H}$,

$$
\mathcal{L}_{+}=\left\{\left(\begin{array}{c}
x \\
K x
\end{array}\right): x \in \mathcal{D}(K)\right\}
$$

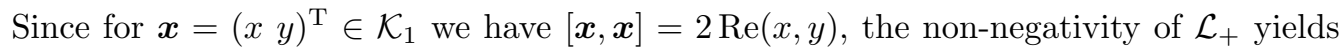
that the operator $K$ is accretive in $\mathcal{H}$, that is, $\operatorname{Re}(K x, x) \geqslant 0, x \in \mathcal{D}(K)$ (see $[\mathbf{2 1}$, Chapter V.3.10]). The fact that the subspace $\mathcal{L}_{+}$is maximal non-negative implies that the operator $K$ in (7.3) is maximal accretive in $\mathcal{H}$, that is, it admits no proper accretive extension.

Remark 7.4. For a general definitizable operator in a Krein space, the maximal nonnegative invariant subspace $\mathcal{L}_{+}$is not uniquely determined, and so we do not have a uniqueness result for $\mathcal{L}_{+}$in Lemma 7.1. However, if $V=0$, uniqueness can be proved and the maximal non-negative invariant subspace is of the form

$$
\mathcal{L}_{+}=\left\{\left(\begin{array}{c}
x \\
H_{0}^{1 / 2} x
\end{array}\right): x \in \mathcal{H}\right\}
$$

which shows that in this case $K=H_{0}^{1 / 2}$.

In the following we consider the operator $K+V$ which is, in general, only quasi-accretive (see [21, Chapter V.3.10]). Therefore, we define

$$
\nu:=\min \left\{0, \inf _{x \neq 0} \frac{(V x, x)}{(x, x)}\right\} \leqslant 0 .
$$

Then the operator $K+V-\nu$ is maximal accretive in $\mathcal{H}$ and thus generates the contractive strongly continuous semi-group

$$
S(\tau):=\mathrm{e}^{-\tau(K+V-\nu)}, \quad \tau \geqslant 0 .
$$

As a consequence, the operator $K+V$ generates the quasi-bounded strongly continuous semi-group

$$
T(\tau):=\mathrm{e}^{-\tau(K+V)}, \quad \tau \geqslant 0,
$$

in $\mathcal{H}$ such that $\|T(\tau)\| \leqslant \mathrm{e}^{\tau \nu}$ (cf. [10, Theorem 3.1]).

The next theorem establishes the existence of solutions of the abstract differential equation (1.14).

Theorem 7.5. Suppose that $V$ is bounded and that the operator $S=V H_{0}^{-1 / 2}$ can be decomposed as $S=S_{0}+S_{1}$ with $\left\|S_{0}\right\|<1$ and $S_{1}$ compact. There then exists a maximal accretive operator $K$ in $\mathcal{H}$ such that, with the semi-group $(T(\tau))_{\tau \geqslant 0}$ given by $T(\tau):=\mathrm{e}^{-\tau(K+V)}, \tau \geqslant 0$, for any initial value $v_{0} \in \mathcal{D}\left((K+V)^{2}\right)$, the function

$$
v(\tau):=T(\tau) v_{0}, \quad \tau \geqslant 0
$$

is a classical solution of the Cauchy problem

$$
\ddot{v}(\tau)+2 V \dot{v}(\tau)+V^{2} v(\tau)-H_{0} v(\tau)=0, \quad \tau \geqslant 0, v(0)=v_{0} .
$$


The spectrum of the infinitesimal generator $K+V$ of the semi-group $(T(\tau))_{\tau \geqslant 0}$ contains the eigenvalues of $A_{1}$ in the open upper half-plane, the spectral points of positive type of $A_{1}$ and the real eigenvalues of $A_{1}$ that are not of negative type.

Proof. By Theorem 5.7 (ii), the non-real spectrum of $A_{1}$ consists only of finitely many points. Thus, we can choose $\beta \in \rho\left(A_{1}\right)$ such that $\operatorname{Re} \beta<\nu$, where $\nu$ is given by (7.4). Then, according to Lemma 7.1 and Remark 7.2, there exists at least one maximal nonnegative subspace $\mathcal{L}_{+}$in $\mathcal{K}_{1}$ such that $\left(A_{1}-\beta\right)^{-1} \mathcal{L}_{+} \subset \mathcal{L}_{+}$, and hence

$$
\mathcal{L}_{+} \subset\left(A_{1}-\beta\right)\left(\mathcal{L}_{+} \cap \mathcal{D}\left(A_{1}\right)\right) .
$$

According to Lemma 7.3 and the remarks following its proof, there exists a maximal accretive operator $K$ in $\mathcal{H}$ so that $\mathcal{L}_{+}$is the graph of $K$, that is, (7.3) holds. For the function $v$ defined in $(7.6)$ we have $v(\tau) \in \mathcal{D}\left((K+V)^{2}\right)$, since $v_{0} \in \mathcal{D}\left((K+V)^{2}\right)$ and thus the derivatives $\dot{v}(\tau), \ddot{v}(\tau)$ exist in the norm topology of $\mathcal{H}$ :

$$
\dot{v}(\tau)=-(K+V) v(\tau), \quad \ddot{v}(\tau)=(K+V)^{2} v(\tau), \quad \tau \geqslant 0 .
$$

We introduce the function

$$
w(\tau):=(K+V-\beta) v(\tau), \quad \tau \geqslant 0 .
$$

Then $w(\tau) \in \mathcal{D}(K+V)=\mathcal{D}(K)$ and $(w(\tau) K w(\tau))^{\mathrm{T}} \in \mathcal{L}_{+}$for all $\tau \geqslant 0$. According to $(7.8)$, there exists an element $(\hat{v}(\tau) K \hat{v}(\tau))^{\mathrm{T}} \in \mathcal{L}_{+} \cap \mathcal{D}\left(A_{1}\right)$ such that

$$
\left(\begin{array}{c}
w(\tau) \\
K w(\tau)
\end{array}\right)=\left(A_{1}-\beta\right)\left(\begin{array}{c}
\hat{v}(\tau) \\
\hat{v}(\tau)
\end{array}\right), \quad \tau \geqslant 0
$$

This equation is equivalent to the system

$$
\begin{aligned}
(K+V-\beta) \hat{v}(\tau) & =w(\tau), \\
\left(H_{0}+(V-\beta) K\right) \hat{v}(\tau) & =K w(\tau)
\end{aligned}
$$

for all $\tau \geqslant 0$. Since $\operatorname{Re} \beta<\nu$ and $K$ is maximal accretive, we have $\beta \in \rho(K+V)$. Thus, (7.11) and (7.10) yield $\hat{v}(\tau)=v(\tau), \tau \geqslant 0$. Now (7.11) and (7.12) imply that

$$
\left(H_{0}+(V-\beta) K\right) v(\tau)=K(K+V-\beta) v(\tau), \quad \tau \geqslant 0,
$$

or, equivalently,

$$
H_{0} v(\tau)+2 V(K+V) v(\tau)-V^{2} v(\tau)=(K+V)^{2} v(\tau), \quad \tau \geqslant 0 .
$$

From this we obtain (7.7) using (7.9).

To prove the last claim, we first consider a point $\lambda_{0}$ that is either an eigenvalue of $A_{1}$ in the open upper half-plane or a real eigenvalue of $A_{1}$ that is not of negative type. In both cases, Lemma 7.1 shows that there exists an eigenvector $\boldsymbol{x}_{0}$ of $A_{1}$ at $\lambda_{0}$ that belongs 
to $\mathcal{L}_{+}$. This means that there exists an $x_{0} \in \mathcal{D}(K)=\mathcal{D}(K+V)$ so that $\boldsymbol{x}_{0}=\left(x_{0} K x_{0}\right)^{\mathrm{T}}$ and

$$
\left(\begin{array}{cc}
V & I \\
H_{0} & V
\end{array}\right)\left(\begin{array}{c}
x_{0} \\
K x_{0}
\end{array}\right)=\lambda_{0}\left(\begin{array}{c}
x_{0} \\
K x_{0}
\end{array}\right) .
$$

Comparing the first components, we find $(K+V) x_{0}=\lambda_{0} x_{0}$, i.e. $\lambda_{0} \in \sigma_{\mathrm{p}}(K+V)$. Finally, we consider a spectral point $\lambda_{0}$ of positive type of $A_{1}$. In this case, there exists a bounded admissible open interval $\Gamma \subset \mathbb{R}$ such that $\lambda_{0} \in \Gamma$ and $E(\Gamma) \mathcal{K}_{1}$ is a positive subspace, which is contained in $\mathcal{L}_{+}$by Lemma 7.1. Then $\lambda_{0}$ is an eigenvalue or approximate eigenvalue of the restriction of $A_{1}$ to $E(\Gamma) \mathcal{K}_{1}$; hence, there exists a sequence $\left(\boldsymbol{x}_{n}\right) \subset E(\Gamma) \mathcal{K}_{1} \subset \mathcal{L}_{+}$such that $\left\|\boldsymbol{x}_{n}\right\|=1$ and $\left(A_{1}-\lambda_{0}\right) \boldsymbol{x}_{n} \rightarrow \mathbf{0}, n \rightarrow \infty$. As above, it is easy to see that, with $\boldsymbol{x}_{n}=\left(x_{n} K x_{n}\right)^{\mathrm{T}}$, we have $\liminf _{n \rightarrow \infty}\left\|x_{n}\right\|>0$ and $(K+V) x_{n}-\lambda_{0} x_{n} \rightarrow 0, n \rightarrow \infty$, and hence $\lambda_{0} \in \sigma(K+V)$.

Remark 7.6. Using the spectral mapping theorem, it can be shown that the spectrum of $K+V$ consists exactly of the points described in the last sentence of the theorem.

Remark 7.7. The function $v(\tau)=T(\tau) v_{0}, \tau \geqslant 0$, is defined for arbitrary elements $v_{0} \in \mathcal{H}$. However, if $H_{0}$ is unbounded, it does not necessarily have a second derivative, and hence $v$ is only a solution in some weaker sense.

Similar considerations as in this section apply to a maximal non-positive invariant subspace of $A_{1}$, which leads to a semi-group and also to a solution of the differential equation (1.14) for $\tau \leqslant 0$.

\section{Application to the Klein-Gordon equation in $\mathbb{R}^{n}$}

In this section we consider the Klein-Gordon equation (1.1) in $\mathbb{R}^{n}$. Here $\mathcal{H}=L_{2}\left(\mathbb{R}^{n}\right)$ with corresponding inner product $(\cdot, \cdot)_{2}$ and norm $\|\cdot\|_{2}, H_{0}=-\Delta+m^{2}$, and $V=e q$ is the maximal multiplication operator by the real-valued measurable function $e q: \mathbb{R}^{n} \rightarrow \mathbb{R}$. In the following we formulate necessary and sufficient conditions for Assumptions 3.1 and 5.1 and we apply the results of the previous sections to the Klein-Gordon equation in $\mathbb{R}^{n}$.

Many different sufficient conditions for the relative boundedness and for the relative compactness of a multiplication operator with respect to $H_{0}^{1 / 2}=\left(-\Delta+m^{2}\right)^{1 / 2}$ have been established (see, for example, $[\mathbf{2 2}, \mathbf{3 9}, \mathbf{4 3}]$ and the more specialized references therein). Examples considered in $[\mathbf{2 7}, \S 6]$ include Rollnik potentials, which are $\left(-\Delta+m^{2}\right)^{1 / 2}$ bounded, and potentials belonging to $L_{p}\left(\mathbb{R}^{n}\right)$ with $n \leqslant p<\infty$, which are $\left(-\Delta+m^{2}\right)^{1 / 2}$ compact. The most general description in terms of necessary and sufficient conditions, which we present here, has been given by Maz'ya and Shaposhnikova in $[\mathbf{3 1}, \mathbf{3 3}]$.

\subsection{Assumptions 3.1 and 5.1}

It is well known (see $[\mathbf{4 4}, \S \S 1.3 .1,1.3 .2])$ that, for $H_{0}=-\Delta+m^{2}$, the spaces $\mathcal{H}_{\alpha}=$ $\mathcal{D}\left(H_{0}^{\alpha}\right), \alpha \in[0,1]$, introduced in (4.1) are the Sobolev spaces of order $2 \alpha$ associated with 
$L_{2}\left(\mathbb{R}^{n}\right)$ (see the definition given below):

$$
\mathcal{H}_{\alpha}=W_{2}^{2 \alpha}\left(\mathbb{R}^{n}\right), \quad \alpha \in[0,1] .
$$

Hence, Assumption 3.1, which reads $\mathcal{H}_{1 / 2}=\mathcal{D}\left(H_{0}^{1 / 2}\right) \subset \mathcal{D}(V)$, now becomes

Assumption 8.1. $W_{2}^{1}\left(\mathbb{R}^{n}\right) \subset \mathcal{D}(V)$.

This is equivalent to $V \in L\left(W_{2}^{1}\left(\mathbb{R}^{n}\right), L_{2}\left(\mathbb{R}^{n}\right)\right)$ or to the fact that $V$ is $\left(-\Delta+m^{2}\right)^{1 / 2}$ bounded; the latter means that there exist constants $a, b \geqslant 0$ such that

$$
\|V u\|_{2} \leqslant a\|u\|_{2}+b\left\|\left(-\Delta+m^{2}\right)^{1 / 2} u\right\|_{2}, \quad u \in W_{2}^{1}\left(\mathbb{R}^{n}\right) .
$$

Therefore, Assumption 5.1 (and hence Assumption 3.1) is satisfied if the restriction of $V$ to $W_{2}^{1}\left(\mathbb{R}^{n}\right)$ can be decomposed as follows.

Assumption 8.2. $V=V_{0}+V_{1}$ such that $V_{0}$ is $\left(-\Delta+m^{2}\right)^{1 / 2}$-bounded and satisfies (8.1) with $a / m+b<1$ and $V_{1}$ is $\left(-\Delta+m^{2}\right)^{1 / 2}$-compact.

Before we formulate abstract necessary and sufficient conditions for Assumptions 8.1 and 8.2, we consider an example for which both assumptions may be checked directly using Hardy's inequality and Sobolev's embedding theorems (see [27, Theorem 6.1, Proposition 6.4 and Example 6.5]).

Example 8.3. Let $n \geqslant 3$. Assumption 8.2 is satisfied for

$$
V(x)=\frac{\gamma}{|x|}+V_{1}(x), \quad x \in \mathbb{R}^{n} \backslash\{0\},
$$

with $\gamma \in \mathbb{R},|\gamma|<(n-2) / 2$, and $V_{1} \in L_{p}\left(\mathbb{R}^{n}\right), n \leqslant p<\infty$.

Instead of the Coulomb part $V_{0}(x)=\gamma /|x|$, we could also assume that $V_{0}$ is bounded, $V_{0} \in L_{\infty}\left(\mathbb{R}^{n}\right)$ with $\left\|V_{0}\right\|_{\infty}<m$, or that $V_{0}^{2}$ is a so-called Rollnik potential (see $[\mathbf{3 9}, \mathbf{4 3}]$ ) with Rollnik norm $\left\|V_{0}^{2}\right\|_{R}<4 \pi$ (see [27, Theorem 6.2]).

Note that the admission of the relatively compact part $V_{1}$ of $V$, which is not subject to any relative norm bound, may give rise to complex eigenvalues, even if $V$ is a bounded potential. This was observed in [42] for potentials represented by a sufficiently deep well.

In general, the property that $V_{0}$ is $\left(-\Delta+m^{2}\right)^{1 / 2}$-bounded means that $V_{0}$ belongs to the space $M\left(W_{2}^{1}\left(\mathbb{R}^{n}\right), L_{2}\left(\mathbb{R}^{n}\right)\right)$ of bounded multipliers from the Sobolev space $W_{2}^{1}\left(\mathbb{R}^{n}\right)$ into $L_{2}\left(\mathbb{R}^{n}\right)$, the property that $V_{1}$ is $\left(-\Delta+m^{2}\right)^{1 / 2}$-compact means that $V_{1}$ belongs to the space $M^{\circ}\left(W_{2}^{1}\left(\mathbb{R}^{n}\right), L_{2}\left(\mathbb{R}^{n}\right)\right)$ of compact multipliers from $W_{2}^{1}\left(\mathbb{R}^{n}\right)$ into $L_{2}\left(\mathbb{R}^{n}\right)$ (see $\left.[\mathbf{3 3}]\right)$. Necessary and sufficient conditions for functions $V$ to belong to a space $M\left(W_{2}^{m}\left(\mathbb{R}^{n}\right), W_{2}^{l}\left(\mathbb{R}^{n}\right)\right)$ of bounded multipliers or the corresponding space of compact multipliers have been established by Maz'ya and Shaposhnikova for integer $m$ and $l$ in [31] and for fractional $m$ and $l$ in $[\mathbf{3 2}]$ (see [33]). In view of Assumption 3.1, we restrict ourselves to the case $l=0$. In the following we introduce all necessary definitions to formulate these criteria in Theorem 8.4, below. 
If $\mathcal{S}_{1}$ and $\mathcal{S}_{2}$ are Banach spaces of functions on $\mathbb{R}^{n}$, then a function $\gamma: \mathcal{S}_{1} \rightarrow \mathcal{S}_{2}$ is called a bounded multiplier from $\mathcal{S}_{1}$ into $\mathcal{S}_{2}, \gamma \in M\left(\mathcal{S}_{1}, \mathcal{S}_{2}\right)$, if

$$
\|\gamma\|_{M\left(\mathcal{S}_{1}, \mathcal{S}_{2}\right)}:=\sup \left\{\|\gamma u\|_{\mathcal{S}_{2}}: u \in \mathcal{S}_{1},\|u\|_{\mathcal{S}_{1}}=1\right\}<\infty .
$$

With any Banach space $\mathcal{S}$ of functions on $\mathbb{R}^{n}$, we associate the space

$$
\mathcal{S}_{\text {loc }}:=\left\{u: \mathbb{R}^{n} \rightarrow \mathbb{C}: \text { for all } \eta \in C_{0}^{\infty}\left(\mathbb{R}^{n}\right) \text { and } \eta u \in \mathcal{S}\right\} .
$$

For $s>0$, we denote by $[s]$ and $\{s\}$ the integer and fractional part, respectively, of $s$, i.e. $s=[s]+\{s\}$ with $[s] \in \mathbb{N}$ and $0 \leqslant\{s\}<1$. For $k \in \mathbb{N}$, we denote by $\nabla_{k}$ the gradient of order $k$, that is,

$$
\nabla_{k}=\left(\frac{\partial^{k}}{\partial x_{1}^{\alpha_{1}} \cdots \partial x_{n}^{\alpha_{n}}}\right)_{\alpha_{1}+\cdots+\alpha_{n}=k} .
$$

For $s>0$ and $1 \leqslant p<\infty$, the fractional derivative $D_{p, s}$ of order $s$ in $L_{p}\left(\mathbb{R}^{n}\right)$ of a function $u$ on $\mathbb{R}^{n}$ is given by

$$
\left(D_{p, s} u\right)(x):=\left(\int_{\mathbb{R}^{n}}\left|\left(\nabla_{[s]} u\right)(x+h)-\left(\nabla_{[s]} u\right)(x)\right||h|^{n-p\{s\}} \mathrm{d} h\right)^{1 / p} .
$$

The fractional Sobolev space $W_{p}^{s}\left(\mathbb{R}^{n}\right)$ is defined as the closure of $C_{0}^{\infty}\left(\mathbb{R}^{n}\right)$ with respect to the norm

$$
\|u\|_{p, s}:=\left\|D_{s, p} u\right\|_{p}+\|u\|_{p}, \quad u \in W_{p}^{s}\left(\mathbb{R}^{n}\right) ;
$$

henceforth $\|\cdot\|_{p}$ denotes the standard norm in $L_{p}\left(\mathbb{R}^{n}\right)$. For integer $s=k \in \mathbb{N}$, the space $W_{p}^{s}\left(\mathbb{R}^{n}\right)$ coincides with the classical Sobolev space

$$
W_{p}^{k}\left(\mathbb{R}^{n}\right):=\left\{u \in L_{p}\left(\mathbb{R}^{n}\right): \nabla_{j} u \in L_{p}\left(\mathbb{R}^{n}\right), j=1,2, \ldots, k\right\}
$$

and the norm (8.2) is equivalent to

$$
\|u\|_{p, k}:=\left(\sum_{j=0}^{k}\left\|\nabla_{j} u\right\|_{p}^{2}\right)^{1 / 2}, \quad u \in W_{p}^{k}\left(\mathbb{R}^{n}\right) .
$$

Finally, for a compact subset $e \subset \mathbb{R}^{n}$, we define the $(p, s)$-capacity of $e$ by

$$
\operatorname{cap}\left(e, W_{p}^{s}\left(\mathbb{R}^{n}\right)\right):=\inf \left\{\|u\|_{p, s}: u \in C_{0}^{\infty}\left(\mathbb{R}^{n}\right),\left.u\right|_{e} \geqslant 1\right\} .
$$

In the following, if $\omega$ varies in some set $\Omega$ and $a, b$ depend on $\omega$, we write $a(\omega) \sim b(\omega)$ if there exist constants $c_{1}, c_{2}>0$ such that $c_{1} b(\omega) \leqslant a(\omega) \leqslant c_{2} b(\omega)$ for all $\omega \in \Omega$.

Theorem 8.4. Let $V: \mathbb{R}^{n} \rightarrow \mathbb{C}$ be a measurable function, $m \in \mathbb{N}$, and $p \in(1, \infty)$. Then $V \in M\left(W_{p}^{m}\left(\mathbb{R}^{n}\right), L_{p}\left(\mathbb{R}^{n}\right)\right.$ ) (the space of bounded multipliers) if and only if there exists a constant $c>0$ such that, for any compact subset $e \subset \mathbb{R}^{n}$,

$$
\|V ; e\|_{p}^{p}:=\int_{e}|V(x)|^{p} \mathrm{~d} x \leqslant c \operatorname{cap}\left(e, W_{p}^{m}\left(\mathbb{R}^{n}\right)\right),
$$


and

$$
\|V\|_{M\left(W_{p}^{m}\left(\mathbb{R}^{n}\right), L_{p}\left(\mathbb{R}^{n}\right)\right)} \sim \sup _{\substack{e \subset \mathbb{R}^{n} \text { compact } \\ \text { diam } e \leqslant 1}} \frac{\|V ; e\|_{p}}{\operatorname{cap}\left(e, W_{p}^{m}\left(\mathbb{R}^{n}\right)\right)^{1 / p}} .
$$

Furthermore, $V \in M^{\circ}\left(W_{p}^{m}\left(\mathbb{R}^{n}\right), L_{p}\left(\mathbb{R}^{n}\right)\right)$ (the space of compact multipliers) if and only if

$$
\lim _{\delta \rightarrow 0} \sup _{\substack{e \in \mathbb{R}^{n} \text { compact, } \\ \text { diam } e \leqslant \delta}} \frac{\|V ; e\|_{p}}{\operatorname{cap}\left(e, W_{p}^{m}\left(\mathbb{R}^{n}\right)\right)^{1 / p}}=0 .
$$

The proof of this theorem may be found in [33, $\S 2.1 .4$ and Lemma 2.2.2/1].

\subsection{Application of Theorems 5.7, 5.9 and 6.5}

If the potential $V$ satisfies Assumption 8.2 (and hence Assumptions 3.1 and 5.1), then all results of the previous sections apply to the Klein-Gordon equation in $\mathbb{R}^{n}$ and we obtain the following statements.

Recall that, by Assumption 8.1, the operator $V$ maps the space $W_{2}^{k}\left(\mathbb{R}^{n}\right)$ boundedly onto $W_{2}^{k-1}\left(\mathbb{R}^{n}\right)$ for all $k \in[0,1]$ (see Remark 4.1, (4.5)).

Theorem 8.5. Suppose that $W_{2}^{1}\left(\mathbb{R}^{n}\right) \subset \mathcal{D}(V)$ and that $V=V_{0}+V_{1}$, where $V_{0}$ is $\left(-\Delta+m^{2}\right)^{1 / 2}$-bounded satisfying (8.1) with $a / m+b<1$ and $V_{1}$ is $\left(-\Delta+m^{2}\right)^{1 / 2}$-compact.

(i) The operator $A_{2}$ given by

$$
\begin{aligned}
\mathcal{D}\left(A_{2}\right) & :=\left\{\left(\begin{array}{l}
x \\
y
\end{array}\right) \in W_{2}^{1 / 2}\left(\mathbb{R}^{n}\right) \oplus W_{2}^{-1 / 2}\left(\mathbb{R}^{n}\right): x \in W_{2}^{1}\left(\mathbb{R}^{n}\right), y \in L_{2}\left(\mathbb{R}^{n}\right),\right. \\
\left.V x+y \in W_{2}^{1 / 2}\left(\mathbb{R}^{n}\right),\left(-\Delta+m^{2}\right) x+V y \in W_{2}^{-1 / 2}\left(\mathbb{R}^{n}\right)\right\} & \\
A_{2}\left(\begin{array}{l}
x \\
y
\end{array}\right): & :\left(\begin{array}{c}
V x+y \\
\left(-\Delta+m^{2}\right) x+V y
\end{array}\right)
\end{aligned}
$$

is a self-adjoint and definitizable operator in the Krein space given by $\mathcal{K}_{2}=$ $W_{2}^{1 / 2}\left(\mathbb{R}^{n}\right) \oplus W_{2}^{-1 / 2}\left(\mathbb{R}^{n}\right)$ with indefinite inner product

$$
\begin{aligned}
& {\left[\boldsymbol{x}, \boldsymbol{x}^{\prime}\right]:=\left(\left(-\Delta+m^{2}\right)^{1 / 4} x,\left(-\Delta+m^{2}\right)^{-1 / 4} y^{\prime}\right)_{2}+\left(\left(-\Delta+m^{2}\right)^{-1 / 4} y,\left(-\Delta+m^{2}\right)^{1 / 4} x^{\prime}\right)_{2},} \\
& \text { for } \boldsymbol{x}=(x y)^{\mathrm{T}}, \boldsymbol{x}^{\prime}=\left(x^{\prime} y^{\prime}\right)^{\mathrm{T}} \in W_{2}^{1 / 2}\left(\mathbb{R}^{n}\right) \oplus W_{2}^{-1 / 2}\left(\mathbb{R}^{n}\right) .
\end{aligned}
$$

(ii) The non-real spectrum of $A_{2}$ is symmetric to the real axis and consists of at most finitely many pairs of eigenvalues $\lambda, \bar{\lambda}$ of finite type; the algebraic eigenspaces corresponding to $\lambda$ and $\bar{\lambda}$ are isomorphic. There are no complex eigenvalues if

$$
\left\|V\left(-\Delta+m^{2}\right)^{-1 / 2}\right\|<1 .
$$

(iii) The essential spectrum of $A_{2}$ is real and has a gap around 0; more precisely,

$$
\sigma_{\text {ess }}\left(A_{2}\right) \subset \mathbb{R} \backslash(-\alpha, \alpha), \quad \alpha:=\left(1-\left(\frac{a}{m}+b\right)\right) m .
$$


(iv) The operator $A_{2}$ is generates a strongly continuous group $\left(\mathrm{e}^{\mathrm{i} t A_{2}}\right)_{t \in \mathbb{R}}$ of unitary operators in the Krein space $\mathcal{K}_{2}=W_{2}^{1 / 2}\left(\mathbb{R}^{n}\right) \oplus W_{2}^{-1 / 2}\left(\mathbb{R}^{n}\right)$.

(v) For every initial value $\boldsymbol{x}_{0} \in \mathcal{D}\left(A_{2}\right)$, the Cauchy problem

$$
\frac{\mathrm{d} \boldsymbol{x}}{\mathrm{d} t}=\mathrm{i} A_{2} \boldsymbol{x}, \quad \boldsymbol{x}(0)=\boldsymbol{x}_{0},
$$

has a unique classical solution $\boldsymbol{x} \in C^{1}\left(\mathbb{R}, W_{2}^{1 / 2}\left(\mathbb{R}^{n}\right) \oplus W_{2}^{-1 / 2}\left(\mathbb{R}^{n}\right)\right)$ given by $\boldsymbol{x}(t)=$ $\mathrm{e}^{\mathrm{i} t A_{2}} \boldsymbol{x}_{0}, t \in \mathbb{R}$.

The Cauchy problem for $A_{2}$ is equivalent to an initial-value problem for the KleinGordon equation (1.1). This leads to the following consequence of Theorem $8.5(\mathrm{v})$.

Theorem 8.6. Suppose that the potential $V$ satisfies the assumptions of Theorem 8.5. Then the initial-value problem

$$
\left(\left(\frac{\partial}{\partial t}-\mathrm{i} e q\right)^{2}-\Delta+m^{2}\right) \psi=0, \quad \psi(\cdot, 0)=\psi_{0}, \quad \frac{\partial \psi}{\partial t}(\cdot, 0)=\psi_{1},
$$

in the space $W_{2}^{-1 / 2}\left(\mathbb{R}^{n}\right)$ has a unique classical solution $\psi_{\mathrm{s}}$ if $\psi_{0} \in W_{2}^{1}\left(\mathbb{R}^{n}\right), \psi_{1} \in$ $W_{2}^{1 / 2}\left(\mathbb{R}^{n}\right)$ and $\left(-\Delta-V^{2}\right) \psi_{0} \in W_{2}^{-1 / 2}\left(\mathbb{R}^{n}\right)$, and the function $t \mapsto \psi_{\mathbf{s}}(\cdot, t)$ belongs to $C^{1}\left(\mathbb{R}, W_{2}^{1 / 2}\left(\mathbb{R}^{n}\right)\right) \cap C^{2}\left(\mathbb{R}, W_{2}^{-1 / 2}\left(\mathbb{R}^{n}\right)\right)$.

Proof. The Cauchy problem for $A_{2}$ arises from (8.3) by means of the substitution

$$
x(t)=\psi(\cdot, t), \quad y(t)=\left(-\mathrm{i} \frac{\partial}{\partial t}-V\right) \psi(\cdot, t), \quad t \in \mathbb{R} ;
$$

hence, the initial value for the Cauchy problem for $A_{2}$ is given by

$$
\boldsymbol{x}_{0}=\left(\begin{array}{l}
x(0) \\
y(0)
\end{array}\right)=\left(\begin{array}{c}
\psi(\cdot, 0) \\
-\mathrm{i} \frac{\partial \psi}{\partial t}(\cdot, 0)-V \psi(\cdot, 0)
\end{array}\right)=\left(\begin{array}{c}
\psi_{0} \\
-\mathrm{i} \psi_{1}-V \psi_{0}
\end{array}\right) .
$$

Since $V$ maps $W_{2}^{1}\left(\mathbb{R}^{n}\right)$ boundedly in $L_{2}\left(\mathbb{R}^{n}\right)$ by Assumption 8.1 , the assumptions on $\psi_{0}$ and $\psi_{1}$ guarantee that $\boldsymbol{x}_{0} \in \mathcal{D}\left(A_{2}\right)$. Hence, Theorem $8.5(\mathrm{v})$ yields a unique solution

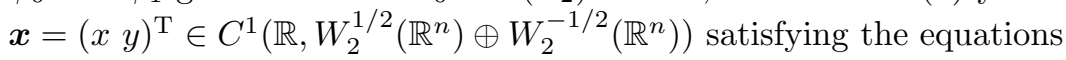

$$
\begin{aligned}
& \frac{\mathrm{d} x}{\mathrm{~d} t}=\mathrm{i}(V x+y) \quad \text { in } W_{2}^{1 / 2}\left(\mathbb{R}^{n}\right), \\
& \frac{\mathrm{d} y}{\mathrm{~d} t}=\mathrm{i}\left(\left(-\Delta+m^{2}\right) x+V y\right) \quad \text { in } W_{2}^{-1 / 2}\left(\mathbb{R}^{n}\right) .
\end{aligned}
$$

Differentiating the first equation and using the second one, we obtain that $x \in$ $C^{1}\left(\mathbb{R}, W_{2}^{1 / 2}\left(\mathbb{R}^{n}\right)\right) \cap C^{2}\left(\mathbb{R}, W_{2}^{-1 / 2}\left(\mathbb{R}^{n}\right)\right)$ and that $x$ satisfies $(8.3)$ in $W_{2}^{-1 / 2}\left(\mathbb{R}^{n}\right)$.

Remark 8.7. If only $\psi_{0} \in W_{2}^{1 / 2}\left(\mathbb{R}^{n}\right)$ and $\psi_{1} \in W_{2}^{-1 / 2}\left(\mathbb{R}^{n}\right)$, then $\boldsymbol{x}_{0} \in W_{2}^{1 / 2}\left(\mathbb{R}^{n}\right) \oplus$ $W_{2}^{-1 / 2}\left(\mathbb{R}^{n}\right)$. In this case we only obtain mild solutions of the Cauchy problem for $A_{2}$ (see Remark 6.6) and thus solutions of (8.3) in some weaker sense. 
If the potential $V$ is $\left(-\Delta+m^{2}\right)^{1 / 2}$-compact, a similar result was proved in $[\mathbf{1 8}, \S 5]$, also using the regularity of the critical point $\infty$ of $A_{2}$.

The above theorem should also be compared with a corresponding result which can be obtained using the operator $A$ introduced in $[\mathbf{2 7}]$ (see $\S 5$ ). Since $A$ is a self-adjoint operator in the Pontryagin space $\mathcal{K}=\mathcal{H}_{1 / 2} \oplus \mathcal{H}=W_{2}^{1}\left(\mathbb{R}^{n}\right) \oplus L_{2}\left(\mathbb{R}^{n}\right)$, it generates a group $\left(\mathrm{e}^{\mathrm{i} t A}\right)_{t \in \mathbb{R}}$ of unitary operators in this space. By means of the substitution

$$
x(t)=\psi(\cdot, t), \quad y(t)=-\mathrm{i} \frac{\partial \psi}{\partial t}(\cdot, t), \quad t \in \mathbb{R},
$$

one can prove an existence and uniqueness result for classical solutions of (8.3) in $L_{2}\left(\mathbb{R}^{n}\right)$. Here, stronger assumptions on the initial values have to be imposed which ensure that

$$
\boldsymbol{x}(0)=\left(\psi_{0}-\mathrm{i} \psi_{1}\right)^{\mathrm{T}} \in \mathcal{D}(A)=\mathcal{D}(H) \oplus \mathcal{D}\left(H_{0}^{1 / 2}\right) \subset W_{2}^{2}\left(\mathbb{R}^{n}\right) \oplus W_{2}^{1}\left(\mathbb{R}^{n}\right)
$$

$\left(H=H_{0}^{1 / 2}\left(I-S^{*} S\right) H_{0}^{1 / 2}\right.$ being the operator associated with the differential expression $\left.-\Delta+V^{2}\right)$.

Remark 8.8. Suppose that the potential $V$ satisfies the assumptions of Theorem 8.5, and that $1 \in \rho\left(S^{*} S\right)$. Then the initial problem (8.3) in $L_{2}\left(\mathbb{R}^{n}\right)$ has a unique classical solution $\psi_{\mathrm{s}}$ if $\psi_{0} \in \mathcal{D}(H) \subset W_{2}^{2}\left(\mathbb{R}^{n}\right), \psi_{1} \in W_{2}^{1}\left(\mathbb{R}^{n}\right)$, and the function $t \mapsto \psi_{\mathrm{s}}(\cdot, t)$ belongs to $C^{1}\left(\mathbb{R}, W_{2}^{1}\left(\mathbb{R}^{n}\right)\right) \cap C^{2}\left(\mathbb{R}, L_{2}\left(\mathbb{R}^{n}\right)\right)$.

This shows that, for smooth initial values as in Remark 8.8, the operator $A$ studied in [27] gives classical solutions of the Klein-Gordon equation (8.3) in $L_{2}\left(\mathbb{R}^{n}\right)$; for less smooth initial values as in Theorem 8.6, the operator $A_{2}$ studied in the present paper still gives classical solutions of the Klein-Gordon equation, but only in $W_{2}^{-1 / 2}\left(\mathbb{R}^{n}\right)$.

Acknowledgements. H.L. and C.T. gratefully acknowledge the support of Deutsche Forschungsgemeinschaft, under Grant no. TR368/6-1. We are grateful to our late colleague Dr Peter Jonas for valuable comments which led to various improvements of this paper; he died on 18 July 2007 shortly before the final version of the manuscript was completed.

\section{References}

1. W. AREndt, Semigroups and evolution equations: functional calculus, regularity and kernel estimates, in Evolutionary equations, Handbook of Differential Equations, Volume I, pp. 1-85 (North-Holland, Amsterdam, 2004).

2. W. Arendt, C. J. K. Batty, M. Hieber and F. Neubrander, Vector-valued Laplace transforms and Cauchy problems, Monographs in Mathematics, Volume 96 (Birkhäuser, Basel, 2001).

3. B. Asmuss, Zur Quantentheorie geladener Klein-Gordon Teilchen in äußeren Feldern, PhD thesis, Hagen, Germany (1990).

4. T. YA. AzIZOV AND I. S. IOKHVIDOV, Linear operators in spaces with an indefinite metric, Pure and Applied Mathematics (Wiley, 1989).

5. A. BACHelot, Superradiance and scattering of the charged Klein-Gordon field by a steplike electrostatic potential, J. Math. Pures Appl. 83 (2004), 1179-1239. 
6. Y. M. Berezansky, Z. G. Sheftel And G. F. Us, Functional analysis, Volume II, Operator Theory, Advances and Applications, Volume 86 (Birkhäuser, Basel, 1996).

7. J. Bognár, Indefinite inner product spaces, Ergebnisse der Mathematik und ihrer Grenzgebiete, Volume 78 (Springer, 1974).

8. B. ĆuRgus, On the regularity of the critical point infinity of definitizable operators, Integ. Eqns Operat. Theory 8 (1985), 462-488.

9. B. ĆURGus And B. NAJMAn, Quasi-uniformly positive operators in Kreı̆n space, in Operator theory and boundary eigenvalue problems, Operator Theory, Advances and Applications, Volume 80, pp. 90-99 (Birkhäuser, Basel, 1995).

10. E. B. Davies, One-parameter semigroups, London Mathematical Society Monographs, Volume 15 (Academic, London, 1980).

11. K.-J. ECKARDT, On the existence of wave operators for the Klein-Gordon equation, Manuscr. Math. 18 (1976), 43-55.

12. K.-J. ECKARDT, Scattering theory for the Klein-Gordon equation, Funct. Approx. Comment. Math. 8 (1980), 13-42.

13. H. Feshbach And F. Villars, Elementary relativistic wave mechanics of spin 0 and spin $\frac{1}{2}$ particles, Rev. Mod. Phys. 30 (1958), 24-45.

14. I. C. Gohberg AND M. G. KREĬN, Introduction to the theory of linear nonselfadjoint operators, Translations of Mathematical Monographs, Volume 18 (American Mathematical Society, Providence, RI, 1969).

15. I. Gohberg, S. Goldberg And M. A. KAAshoek, Classes of linear operators, Volume I, Operator Theory, Advances and Applications, Volume 49 (Birkhäuser, Basel, 1990).

16. P. JonAs, On local wave operators for definitizable operators in Krein space and on a paper by T. Kako, preprint P-46/79 (Zentralinstitut für Mathematik und Mechanik der AdW DDR, Berlin, 1979).

17. P. JonAs, On a problem of the perturbation theory of selfadjoint operators in Kre spaces, J. Operat. Theory 25 (1991), 183-211.

18. P. JonAs, On the spectral theory of operators associated with perturbed Klein-Gordon and wave type equations, J. Operat. Theory 29 (1993), 207-224.

19. P. JonAs, On bounded perturbations of operators of Klein-Gordon type, Glas. Mat. Ser. III 35 (2000), 59-74.

20. T. KAKO, Spectral and scattering theory for the $J$-selfadjoint operators associated with the perturbed Klein-Gordon type equations, J. Fac. Sci. Univ. Tokyo (1) A 23 (1976), 199-221.

21. T. KATO, Perturbation theory for linear operators, Die Grundlehren der mathematischen Wissenschaften, Volume 132 (Springer, 1966).

22. T. KATO, A short introduction to perturbation theory for linear operators (Springer, 1982).

23. S. G. KRĔN, Linear differential equations in Banach space, Translations of Mathematical Monographs, Volume 29 (American Mathematical Society, Providence, RI, 1971).

24. H. LANGER, Invariante Teilräume definisierbarer $J$-selbstadjungierter Operatoren, $A n$ nales Acad. Sci. Fenn. A 475 (1971), 1-23.

25. H. LANGER, Spectral functions of definitizable operators in Kreŭn spaces, in Functional analysis, Lecture Notes in Mathematics, Volume 948, pp. 1-46 (Springer, 1982).

26. H. LANGer AND B. NAJMAn, A Krein space approach to the Klein-Gordon equation, unpublished manuscript (1996).

27. H. LAnger, B. NAJMan And C. Tretter, Spectral theory of the Klein-Gordon equation in Pontryagin spaces, Commun. Math. Phys. 267 (2006), 159-180.

28. L.-E. LUNDBERG, Relativistic quantum theory for charged spinless particles in external vector fields, Commun. Math. Phys. 31 (1973), 295-316.

29. L.-E. LundBerg, Spectral and scattering theory for the Klein-Gordon equation, Commun. Math. Phys. 31 (1973), 243-257. 
30. A. S. MARKUS, Introduction to the spectral theory of polynomial operator pencils, Translations of Mathematical Monographs, Volume 71 (American Mathematical Society, Providence, RI, 1988).

31. V. G. MAZ'YA AND T. O. Shaposhnikova, Multipliers in pairs of spaces of potentials, Math. Nachr. 99 (1980), 363-379.

32. V. G. MAZ'Y Ya And T. O. Shaposhnikova, Multipliers in pairs of spaces of differentiable functions, Trudy Mosk. Mat. Obshc. 43 (1981), 37-80 (in Russian).

33. V. G. MAZ'YA AND T. O. ShapOSHnIKOVA, Theory of multipliers in spaces of differentiable functions, Monographs and Studies in Mathematics, Volume 23 (Pitman, Boston, MA, 1985).

34. B. NAJMAn, Solution of a differential equation in a scale of spaces, Glas. Mat. Ser. III 14 (1979), 119-127.

35. B. Najman, Spectral properties of the operators of Klein-Gordon type, Glas. Mat. Ser. III 15 (1980), 97-112.

36. B. NAJMAn, Localization of the critical points of Klein-Gordon type operators, Math. Nachr. 99 (1980), 33-42.

37. B. Najman, Eigenvalues of the Klein-Gordon equation, Proc. Edinb. Math. Soc. 26 (1983), 181-190.

38. J. PALmer, Symplectic groups and the Klein-Gordon field, J. Funct. Analysis 27 (1978), 308-336.

39. M. REed AND B. Simon, Methods of modern mathematical physics, II, Fourier analysis, self-adjointness (Academic, 1975).

40. M. REED AND B. Simon, Methods of modern mathematical physics, IV, Analysis of operators (Academic/Harcourt Brace, 1978).

41. M. Schechter, The Klein-Gordon equation and scattering theory, Annals Phys. 101 (1976), 601-609.

42. L. I. Schiff, H. SNyder And J. Weinberg, On the existence of stationary states of the mesotron field, Phys. Rev. 57 (1940), 315-318.

43. B. Simon, Quantum mechanics for Hamiltonians defined as quadratic forms, Princeton Series in Physics (Princeton University Press, 1971).

44. H. TRIEBEL, Theory of function spaces, II, Monographs in Mathematics, Volume 84 (Birkhäuser, Basel, 1992).

45. K. Veselić, A spectral theory for the Klein-Gordon equation with an external electrostatic potential, Nucl. Phys. A 147 (1970), 215-224.

46. K. VESELIĆ, On spectral properties of a class of $J$-selfadjoint operators, I, Glas. Mat. Ser. III 7 (1972), 229-248.

47. K. Veselić, On spectral properties of a class of $J$-selfadjoint operators, II, Glas. Mat. Ser. III 7 (1972), 249-254.

48. K. VESELIĆ, A spectral theory of the Klein-Gordon equation involving a homogeneous electric field, J. Operat. Theory 25 (1991), 319-330.

49. R. WEDER, Selfadjointness and invariance of the essential spectrum for the Klein-Gordon equation, Helv. Phys. Acta 50 (1977), 105-115.

50. R. WEDER, Scattering theory for the Klein-Gordon equation, J. Funct. Analysis 27 (1978), 100-117.

51. J. Weidmann, Linear operators in Hilbert spaces, Graduate Texts in Mathematics, Volume 68 (Springer, 1980). 